$\mathrm{MAN} / \mathrm{HEP} / 2014 / 18$

December 2014

\title{
Matter Quantum Corrections to the Graviton Self-Energy and the Newtonian Potential
}

\author{
Daniel Burns* and Apostolos Pilaftsis \\ Consortium for Fundamental Physics, School of Physics and Astronomy, \\ University of Manchester, Manchester M13 9PL, United Kingdom
}

\begin{abstract}
We revisit the calculation of matter quantum effects on the graviton self-energy on a flat Minkowski background, with the aim to acquire a deeper understanding of the mechanism that renders the graviton massless. To this end, we derive a low-energy theorem which directly relates the radiative corrections of the cosmological constant to those of the graviton mass to all orders in perturbation theory. As an illustrative example, we consider an Abelian Higgs model with minimal coupling to gravity and show explicitly how a suitable renormalization of the cosmological constant leads to the vanishing of the graviton mass at the one-loop level. In the same Abelian Higgs model, we also calculate the matter quantum corrections to the Newtonian potential and present analytical formulae in terms of modified Bessel and Struve functions of the particle masses in the loop. We show that the correction to the Newtonian potential exhibits an exponential fall-off dependence on the distance $r$, once the non-relativistic limit with respect to the non-zero loop mass is carefully considered. For massless scalars, fermions and gauge bosons in the loops, we recover the well-known results presented in the literature.
\end{abstract}

\footnotetext{
* daniel.burns@hep.manchester.ac.uk
}

$\dagger$ apostolos.pilaftsis@manchester.ac.uk 


\section{INTRODUCTION}

Symmetries play an instrumental role in quantum field theory to ensure that massless particles at the classical level remain massless against quantum loop effects. For instance, massless vector bosons in Yang-Mills theories stay massless, as a consequence of the gauge symmetry of the effective action. This fact can be understood more easily within the gauge-invariant framework of the background field method [1, 2, in which a non-zero mass for the background Yang-Mills vector boson is forbidden to all orders in perturbation theory. Likewise, massless fermions can be protected from receiving a non-zero mass due to chiral symmetry [3]. Scalar particles can also stay massless to all orders, as a result of symmetries. For example, massless scalar particles could result from the spontaneous breakdown of a global Goldstone symmetry [4]. Other potential symmetries leading to massless scalar particles are supersymmetry [5, 6] or classical scaling (conformal) symmetries [7, 8. Such symmetries have been extensively discussed within the context of a related problem in the Standard Model (SM), the so-called gauge-hierarchy problem [9 11.

The aim of the present paper is to shed light on the mechanism that protects the spin-2 graviton from receiving a non-zero mass beyond the tree level. In this context, we should mention that matter contributions to the graviton self-energy have already been studied in the past to a great extent [12 16. However, in our opinion, the actual mechanism that lies behind the masslessness of the graviton has not yet been adequately elucidated. In particular, a radiatively generated graviton mass will affect the scattering of two scalar fields beyond the tree level. Such calculations are relevant to the study of the quantum corrected Newtonian potential and may be in conflict with well-established observations. It is therefore important to state here that the gauge or diffeomorphisms invariance of the effective action, even within the linearized framework of perturbative quantum gravity (PQG), is not sufficient by itself to guarantee that the graviton remains massless against quantum loop corrections. Specifically, the cosmological constant term is invariant under diffeomorphisms and contains a mass term for the graviton. At the tree level, this problem is resolved (see, e.g. [17) after imposing the equations of motion with respect to the background graviton field, with the aid of which a would-be graviton mass can be removed. Beyond the tree approximation, however, the masslessness of the graviton is not an obvious property, as this problem becomes strongly interrelated with the renormalization of the cosmological constant $\Lambda$.

In quantum field theory, the pole position of a particle propagator encodes all the information about the mass of the particle. As we will show in this paper, the cosmological constant $\Lambda$ plays an important role, as it receives radiative corrections independently of the graviton propagator. These corrections are divergent and must be renormalized, or otherwise naturally suppressed, to give the small value of $\Lambda$ that we observe in the present epoch [18, 19. Upon suitable renormalization of the cosmological constant $\Lambda$ to a flat (Minkowski) background metric, the generated counterterm (CT) $\delta \Lambda$ enters the graviton self-energy explicitly within our linearized framework of PQG. We find that the masslessness property of the graviton is protected by a shift symmetry which is present in any diffeomorphisms invariant theory described by a flat background metric. The absence of the graviton mass will be explicitly demonstrated at the one-loop level in PQG within the context of an Abelian Higgs model.

Given that the framework of PQG is non-renormalizable 20 23, we follow the general lore and treat General Relativity (GR) as an effective field theory [24 with a characteristic ultra-violet (UV) scale equal to the Planck mass $M_{\mathrm{P}}$. Much work has been done within this effective field-theoretic framework, including PQG corrections to the Newtonian and Coulomb potentials, as well as one-loop calculations of graviton-mediated scatterings between matter fields in the non-relativistic limit 24 33. Taking into account the contributions from the graviton and from massless fields of different spin, the established analytic result for the Newtonian potential $V(r)$, between two masses $m_{1}$ and $m_{2}$ being at distance $r$ apart, may be cast into the form [26, 30, 34, 35]:

$$
V(r)=-\frac{G m_{1} m_{2}}{r}\left[1+3 \frac{G\left(m_{1}+m_{2}\right)}{r c^{2}}+\frac{41 \hbar G}{10 \pi c^{3} r^{2}}+\left(\frac{9}{4} N_{0}+3 N_{\frac{1}{2}}+12 N_{1}\right) \frac{\hbar G}{45 \pi c^{3} r^{2}}+O\left(\hbar^{2}\right)\right]
$$

where $G=\hbar c / M_{\mathrm{P}}^{2}$ is Newton's constant and $N_{s}$ is the number of fields with spin $s=0$ (scalar), $\frac{1}{2}$ (Weyl fermion), 1 (vector boson) in units of $\hbar$. Note that the first two terms in (I.1) correspond to the classical and quantum graviton contributions to the Newtonian potential $V(r)$, respectively. The leading radiative corrections to $V(r)$ come from the so-called non-analytic parts of the amplitude, which diverge in the infra-red (IR) limit of vanishing 3-momenta for the external gravitationally-scattered fields. Using a similar approach, we compute the general matter loop corrections to the graviton propagator, as well as the modifications to the Newtonian potential $V(r)$. The matter contributions to $V(r)$ at the one-loop level effect only the graviton self-energy in a generic $2 \rightarrow 2$ scattering process. Thus, we shall show that the contributions of massive matter fields to the resummed graviton self-energies become relevant in the non-relativistic limit and therefore contribute to the Newtonian potential.

The layout of the paper is as follows. After this introductory section, Section II presents a gauged Abelian Higgs model with minimal coupling to gravity. This model serves as an illustrative example, which will help us to define our theoretical PQG framework that can include scalars, fermions and spin-1 fields. Based on this framework, we discuss 
the properties of the corresponding diffeomorphically invariant path integral for the gauged Abelian Higgs model. Given that the model has no gravitational anomalies [36, we derive the master Ward identity (WI) associated with the invariance of the path integral under diffeomorphisms.

In Section III we study the minimization conditions pertinent to the one-loop effective action, where the renormalization of the cosmological constant $\Lambda$ plays a key role to the renormalization of the graviton tadpole graphs. To further illuminate this deep connection, we derive a low-energy theorem that involves graviton correlation functions to all orders in perturbation theory. This Graviton Low Energy Theorem (GLET) may also be utilized to obtain a non-perturbative relation between the tadpole contributions and the graviton self-energy at zero external momentum.

In Section IV] we calculate the matter contributions to the graviton self-energy for the gauged Abelian Higgs model with minimal coupling to gravity. To deal with UV infinities, we adopt the method of dimensional regularisation [37. We then proceed to renormalize the massive matter-field contributions to the graviton self-energy, after properly including the cosmological constant $\mathrm{CT} \delta \Lambda$, as well as higher-dimensional Planck-suppressed operators of the Riemann tensor. We thus show that the graviton field acquires no mass at the one-loop level. We explicitly demonstrate how this result persists to all orders, as a consequence of the GLET and the WI due to invariance of the path integral under diffeomorphisms.

In Section [V] we first review the tree-level calculation for the gravitationally mediated scattering process between two scalar fields, where the classical part of the Newtonian potential $V(r)$ is recovered. We then incorporate the self-energy contributions to the graviton propagator, which is used to determine the matter quantum corrections to the Newtonian potential. Our analytic results are expressed in terms of modified Bessel and Struve functions of the particle masses in the loop. In the massless limit of the loop masses, we reproduce the analytic result given in (I.1), for particles with spin $s=0, \frac{1}{2}, 1$. In the same section, we comment on the independence of $V(r)$ on the gravitational gauge-fixing parameters, as well as on gauge-fixing parameters due to gauge bosons in the loop. Section VI]summarizes our conclusions. Finally, relevant Feynman rules and other technical details that were useful in our computations have been presented in Appendix A.

\section{THEORETICAL FRAMEWORK OF QUANTUM GRAVITY}

In this section, we first outline our theoretical framework within the context of an Abelian Higgs model with minimal coupling to gravity, by making use of the background field method. We then write down the generating functional for this model and discuss its invariance under transformations of diffeomorphism. From the latter, we derive a master WI for diffeomorpshims, which gives rise to an important WI that relates the graviton self-energy to the graviton tadpole graphs to all orders in perturbation theory.

To begin with, we write down the action $S$ of an Abelian Higgs model minimally coupled to gravity as a sum of two terms:

$$
S=S_{G}+S_{M}=\int d^{4} x \sqrt{-g}\left(\Lambda+\frac{1}{\kappa^{2}} R+\mathcal{L}_{M}\right),
$$

where $S_{G}$ is the Hilbert-Einstein action of gravity with a cosmological constant $\Lambda$ and $S_{M} \equiv \int d^{4} x \sqrt{-g} \mathcal{L}_{M}$ is the part of the action that only contains the matter Lagrangian $\mathcal{L}_{M}$. In addition, we denote with $g_{\mu \nu}$ the global metric of the space and $g \equiv \operatorname{det} g_{\mu \nu}$, whilst our convention for the Minkowski metric $\eta_{\mu \nu}$ is $\eta_{\mu \nu}=\operatorname{diag}(1,-1,-1,-1)$. In (II.1), $R$ is the Ricci scalar and $\kappa$ a gravitational coupling constant, which is related to Newton's constant $G$ by $\kappa^{2}=16 \pi G$.

The matter action $S_{M}$ describes a gauged Abelian Higgs model based on the gauge group $U(1)_{Y}$, which realizes spontaneous symmetry breaking. In detail, the matter action $S_{M}$ is given by

$$
S_{M}=\int d^{4} x \sqrt{-g}\left[-\frac{1}{4} g^{\mu \rho} g^{\nu \sigma} F_{\mu \nu} F_{\rho \sigma}+g^{\mu \nu}\left(\nabla_{\mu} \phi\right)^{\dagger} \nabla_{\nu} \phi-\lambda\left(\phi^{\dagger} \phi-\frac{\mu^{2}}{2 \lambda}\right)^{2}\right]+S_{f},
$$

where $S_{f}$ is the fermionic sector of the model, $F_{\mu \nu} \equiv \partial_{\mu} \mathcal{A}_{\nu}-\partial_{\nu} \mathcal{A}_{\mu}$ is the field strength tensor associated with the gauge field $\mathcal{A}_{\mu}$, and $\phi=\frac{1}{\sqrt{2}}(v+\mathcal{H}+i \mathcal{G})$ is a complex scalar field with hypercharge $Y_{\phi}=1$. Moreover, $\nabla_{\mu}$ is the covariant derivative with respect to both the gauge group and the group of diffeomorphisms. Thus, for the scalar field $\phi$, the covariant derivative is simply given by $\nabla_{\mu} \phi=\partial_{\mu} \phi-i e \mathcal{A}_{\mu} \phi$. Here, we follow the standard procedure of general covariantization, namely by first writing down the matter Lagrangian $\mathcal{L}_{M}$ in flat space and then making the substitution $\eta_{\mu \nu} \rightarrow g_{\mu \nu}$ and $\partial_{\mu} \rightarrow \nabla_{\mu}$. In [II.2, we have also included an overall factor $\sqrt{-g}$, so as to get a fully frame-independent action.

Adopting the background field method (BFM), we decompose the fields into background and quantum fields as follows:

$$
\mathcal{H}=\bar{H}+H^{Q}, \quad \mathcal{G}=\bar{G}+G^{Q}, \quad \mathcal{A}_{\mu}=\bar{A}_{\mu}+A_{\mu}^{Q},
$$


where an overbar denotes a background field, whilst a superscript $Q$ denotes a quantum field. The Higgs mechanism will generate a mass $m_{A}$ to the gauge field in the broken phase, given by $m_{A}=e v$, as well as a mass for the Higgs field itself determined through the relation: $m_{H}^{2}=2 \lambda v^{2}$.

The fermionic part $S_{f}$ in (II.2) of the matter action may contain left- and right-handed chiral fermions. For simplicity, we assume one Dirac fermion $\psi$ with hypercharge quantum number $Y_{\psi}=1$, with vector-like couplings to the $U(1)_{Y}$ gauge bosons. This simple setup is also free of chiral anomalies [38, 39. In curved spacetime, spinors have non-trivial transformation properties under the group of diffeomorphisms, which is usually accounted for by the spin connection. Hence, with the inclusion of the Dirac fermion field $\psi=\psi^{Q}$, the fermionic part of the action $S_{f}$ reads:

$$
\mathcal{S}_{f}=\int d^{4} x \sqrt{-g}\left[\frac{1}{2}\left(\nabla_{\mu} \bar{\psi}^{Q}\right) i e_{a}^{\mu} \gamma^{a} \psi^{Q}-\frac{1}{2} \bar{\psi}^{Q} i e_{a}^{\mu} \gamma^{a}\left(\nabla_{\mu} \psi^{Q}\right)-m_{\psi} \bar{\psi}^{Q} \psi^{Q}\right],
$$

where the covariant derivative acting on $\psi$ is given by

$$
\nabla_{\mu} \psi^{Q}=\partial_{\mu} \psi^{Q}-\omega_{\mu}^{a b} \sigma_{a b} \psi^{Q}-i e \mathcal{A}_{\mu}^{Q} \psi^{Q}
$$

In the above, $\sigma_{a b}=\frac{1}{4}\left[\gamma_{a}, \gamma_{b}\right]$ are the Lorentz-group generators in the spinorial representation, $\omega_{\mu}^{a b}$ is the spin connection, which is determined by means of the vielbeins $e_{\mu}^{a}$ as follows:

$$
\omega_{\mu}^{a b}=-g^{\nu \lambda} e_{\lambda}^{a}\left(\partial_{\mu} e_{\nu}^{b}-e_{\sigma}^{b} \Gamma_{\mu \nu}^{\sigma}\right)
$$

Note that the vielbein fields $e_{\mu}^{a}$ are defined through the relations:

$$
g_{\mu \nu} \equiv e_{\mu}^{a} e_{\nu}^{b} \eta_{a b}, \quad e_{\mu}^{a} e_{b}^{\mu}=\delta_{b}^{a}, \quad e_{\mu}^{a} e_{a}^{\nu}=\delta_{\nu}^{\mu},
$$

where the Latin indices $a, b$ etc. refer to the tangent space of the curved spacetime which is locally flat.

To quantise gravity within the BFM framework, we decompose the metric $g_{\mu \nu}$ as

$$
g_{\mu \nu}=\eta_{\mu \nu}+\kappa\left(\bar{h}_{\mu \nu}+h_{\mu \nu}^{Q}\right)=\bar{g}_{\mu \nu}+\kappa h_{\mu \nu}^{Q},
$$

where $h_{\mu \nu}^{Q}$ is the quantum fluctuation of the metric, $\bar{h}_{\mu \nu}$ is the background field and $\bar{g}_{\mu \nu}=\eta_{\mu \nu}+\kappa \bar{h}_{\mu \nu}$. In the absence of a classical gravitational field $\bar{h}_{\mu \nu}$, we have $\bar{g}_{\mu \nu}=\eta_{\mu \nu}$ and the curved space reduces to a Minkowski flat space in this case. In this paper, we will consider a flat background to carry out perturbative calculations within the framework of linearized quantum gravity.

To eliminate the degeneracy in the field space due to the symmetry of diffeomorphisms, we use the gauge fixing condition

$$
G_{a}=(-\bar{g})^{\frac{1}{4}}\left[\bar{g}^{\alpha \beta}\left(\bar{\nabla}_{\alpha} h_{\beta \mu}^{Q}-\sigma \bar{\nabla}_{\mu} h_{\alpha \beta}^{Q}\right)\right] \bar{e}_{a}^{\mu}=\omega_{a}
$$

where $\omega_{a}(x)$ is an arbitrary function and $\bar{e}_{a}^{\mu}$ is the background vielbein field. Employing the Faddeev-Popov gauge fixing procedure, we introduce the gauge-fixing action

$$
S_{\mathrm{GF}, \mathrm{Diff}}=-\frac{1}{2 \xi_{D}} \int d^{4} x \sqrt{-\bar{g}} \bar{g}^{\mu \nu}\left[\bar{g}^{\alpha \beta}\left(\bar{\nabla}_{\alpha} h_{\beta \mu}^{Q}-\sigma \bar{\nabla}_{\mu} h_{\alpha \beta}^{Q}\right)\right]\left[\bar{g}^{\delta \gamma}\left(\bar{\nabla}_{\delta} h_{\gamma \nu}^{Q}-\sigma \bar{\nabla}_{\nu} h_{\delta \gamma}^{Q}\right)\right],
$$

which in turn induces the ghost action

$$
S_{\mathrm{Gh}, \mathrm{Diff}}=-\int d^{4} x \sqrt{-\bar{g}} \bar{\eta}^{\mu}\left(\bar{g}^{\alpha \beta} \bar{\nabla}_{\alpha} \bar{\nabla}_{\beta} \eta_{\mu}+\bar{g}^{\alpha \beta} \bar{R}_{\mu \alpha} \eta_{\beta}+(1-2 \sigma) \bar{g}^{\alpha \beta} \bar{\nabla}_{\mu} \bar{\nabla}_{\alpha} \eta_{\beta}\right)
$$

where $\eta_{\mu}$ and $\bar{\eta}_{\nu}$ are the ghost vector fields associated with the graviton field $h_{\mu \nu}$.

In addition to the diffeomorphisms group, we must also gauge-fix the $U(1)_{Y}$ gauge group. To this end, we consider the gauge fixing term

$$
S_{\mathrm{GF}, \mathrm{U}(1)}=-\frac{1}{2 \xi_{G}} \int d^{4} x \sqrt{-g}\left[g^{\mu \nu} \nabla_{\mu} A_{\nu}^{Q}+e \xi_{G} G^{Q}\left(v+H^{Q}\right)\right]^{2},
$$

which has the property of preserving general covariance whilst breaking the invariance of the gauge group. It also preserves the Higgs-boson low-energy theorem (HLET) 40 44] in its canonical form [45]. The gauge-fixing action $S_{\mathrm{GF}, \mathrm{U}(1)}$ also induces a Faddeev-Popov ghost action, which is given by

$$
S_{\mathrm{Gh}, \mathrm{U}(1)}=-\int d^{4} x \sqrt{-g} \bar{c}\left\{g^{\mu \nu} \nabla_{\mu} \nabla_{\nu}+\frac{e^{2}}{2} \xi_{G}\left[\left(v+H^{Q}\right)^{2}-\left(G^{Q}\right)^{2}\right]\right\} c
$$

where $c, \bar{c}$ are the $U(1)_{Y}$ Faddeev-Popov ghosts. Note that the scalar ghosts $c, \bar{c}$ and their vector counterparts $\eta_{\mu}, \bar{\eta}_{\nu}$ are all anti-commuting negative norm fields. 


\section{II.1. The Diffeomorphically Invariant Path Integral}

To quantize the Abelian Higgs model with minimal coupling to gravity, we introduce the generating functional

$$
\begin{aligned}
Z\left[\bar{h}_{\mu \nu}, \bar{H}, \bar{G}, \bar{A}_{\mu}, J_{h}^{\mu \nu}, J_{\psi}, \bar{J}_{\psi}, J_{H}, J_{G}, J_{A}^{\mu}\right]= & N \int \mathcal{D} \Phi \exp \left[i S\left[\bar{h}_{\mu \nu}, h_{\mu \nu}^{Q}, \mathcal{H}, \mathcal{G}, \psi, \bar{\psi}, \mathcal{A}_{\mu}\right]\right. \\
& \left.+\int d^{4} x \sqrt{-\bar{g}}\left(J_{h}^{\mu \nu} h_{\mu \nu}^{Q}+\bar{J}_{\psi} \psi^{Q}+\bar{\psi}^{Q} J_{\psi}+J_{H} H^{Q}+J_{G} G^{Q}+J_{A}^{\mu} A_{\mu}^{Q}\right)\right]
\end{aligned}
$$

where $N$ is an unphysical overall normalization constant and

$$
\mathcal{D} \Phi \equiv \mathcal{D} h_{\mu \nu}^{Q} \mathcal{D} A_{\mu}^{Q} \mathcal{D} H^{Q} \mathcal{D} G^{Q} \mathcal{D} \bar{\psi}^{Q} \mathcal{D} \psi^{Q}
$$

is a short-hand notation for the integral measure. Under infinitesimal diffeomorphisms, $x^{\mu} \rightarrow x^{\prime \mu}=x^{\mu}+\kappa \epsilon^{\mu}(x)$ with $\epsilon^{\mu}(x) \ll 1$, the action $S$ of the theory remains invariant provided the fields transform as follows:

$$
\begin{aligned}
g_{\mu \nu}^{\prime} & =g_{\mu \nu}+\kappa\left(g_{\nu}^{\alpha} \partial_{\mu} \epsilon_{\alpha}+g_{\mu}^{\alpha} \partial_{\nu} \epsilon_{\alpha}+\epsilon_{\alpha} \partial^{\alpha} g_{\mu \nu}\right), \\
\mathcal{H}^{\prime} & =\mathcal{H}+\kappa \epsilon^{\alpha} \partial_{\alpha} \mathcal{H} \\
\mathcal{G}^{\prime} & =\mathcal{G}+\kappa \epsilon^{\alpha} \partial_{\alpha} \mathcal{G} \\
\psi^{\prime Q} & =\psi^{Q}+\kappa \epsilon^{\alpha} \partial_{\alpha} \psi^{Q} \\
\bar{\psi}^{\prime Q} & =\bar{\psi}^{Q}+\kappa \epsilon^{\alpha} \partial_{\alpha} \bar{\psi}^{Q} \\
\mathcal{A}_{\mu}^{\prime} & =\mathcal{A}_{\mu}+\kappa \epsilon^{\alpha} \partial_{\alpha} \mathcal{A}_{\mu}+\kappa\left(\partial_{\mu} \epsilon^{\alpha}\right) \mathcal{A}_{\alpha} .
\end{aligned}
$$

There is now a degree of arbitrariness in the way the transformations are attributed separately for the background and quantum fields, within the context of the BFM. We choose to distribute the metric transformation as

$$
\begin{aligned}
\bar{h}_{\mu \nu}^{\prime} & =\bar{h}_{\mu \nu}+\partial_{\mu} \epsilon_{\nu}+\partial_{\nu} \epsilon_{\mu}+\kappa\left(\bar{h}_{\nu}^{\alpha} \partial_{\mu} \epsilon_{\alpha}+\bar{h}_{\mu}^{\alpha} \partial_{\nu} \epsilon_{\alpha}+\epsilon_{\alpha} \partial^{\alpha} \bar{h}_{\mu \nu}\right), \\
h_{\mu \nu}^{\prime Q} & =h_{\mu \nu}^{Q}+\kappa\left(h_{\alpha \nu}^{Q} \partial_{\mu} \epsilon^{\alpha}+h_{\alpha \mu}^{Q} \partial_{\nu} \epsilon^{\alpha}+\epsilon_{\alpha} \partial^{\alpha} h_{\mu \nu}^{Q}\right) .
\end{aligned}
$$

Similarly, we distribute the transformations of the $\mathcal{H}, \mathcal{G}$ and $\mathcal{A}_{\mu}$ fields as

$$
\begin{aligned}
\bar{H}^{\prime} & =\bar{H}+\kappa \epsilon^{\alpha} \partial_{\alpha} \bar{H}, & H^{\prime Q} & =H^{Q}+\kappa \epsilon^{\alpha} \partial_{\alpha} H^{Q}, \\
\bar{G}^{\prime} & =\bar{G}+\kappa \epsilon^{\alpha} \partial_{\alpha} \bar{G}, & G^{Q} & =G^{Q}+\kappa \epsilon^{\alpha} \partial_{\alpha} G^{Q} \\
\bar{A}_{\mu}^{\prime} & =\bar{A}_{\mu}+\kappa \epsilon^{\alpha} \partial_{\alpha} \bar{A}_{\mu}+\kappa\left(\partial_{\mu} \epsilon^{\alpha}\right) \bar{A}_{\alpha}, & A_{\mu}^{\prime Q} & =A_{\mu}^{Q}+\kappa \epsilon^{\alpha} \partial_{\alpha} A_{\mu}^{Q}+\kappa\left(\partial_{\mu} \epsilon^{\alpha}\right) A_{\alpha}^{Q} .
\end{aligned}
$$

It is now crucial to check whether the symmetry transformations in II.16 for the action $S$ of the theory leave the integral measure $\mathcal{D} \Phi$ invariant as well. For this purpose, we need to calculate the Jacobian determinant associated with the transformations of diffeomorphism, i.e.

$$
J[\epsilon] \equiv \operatorname{det}\left(\frac{\delta \Phi_{i}^{\prime}(x)}{\delta \Phi_{j}(y)}\right)
$$

where $\Phi_{i} \in\left\{h_{\mu \nu}^{Q}, H^{Q}, G^{Q}, \psi^{Q}, \bar{\psi}^{Q}, A_{\mu}^{Q}\right\}$. Using the fact that

$$
\operatorname{det}(I+A)=1+\operatorname{Tr}(A)+O\left(A^{2}\right)
$$

for small $A$, we obtain that

$$
\begin{aligned}
\operatorname{det}\left(\frac{\delta H^{\prime Q}(x)}{\delta H^{Q}(y)}\right) & =1-\frac{1}{2} \kappa \delta(0) \int d^{4} x \partial_{\mu} \epsilon^{\mu}(x), \\
\operatorname{det}\left(\frac{\delta \psi^{\prime Q}(x)}{\delta \psi^{Q}(y)}\right) & =1-\frac{1}{2} \kappa \delta(0) \int d^{4} x \partial_{\mu} \epsilon^{\mu}(x), \\
\operatorname{det}\left(\frac{\delta A_{\mu}^{\prime Q}(x)}{\delta A_{\nu}^{Q}(y)}\right) & =1-\kappa \delta(0) \int d^{4} x \partial_{\mu} \epsilon^{\mu}(x), \\
\operatorname{det}\left(\frac{\delta h_{\mu \nu}^{\prime Q}(x)}{\delta h_{\rho \sigma}^{Q}(y)}\right) & =1
\end{aligned}
$$


Consequently, for scalars, fermions and spin-1 fields, there seems to be a deviation from 1 . However, one may observe that the integral appearing in the measure's transformation actually vanishes,

$$
\int d^{4} x \partial_{\mu} \epsilon^{\mu}=0
$$

since fields (as well as gauge transformed fields) are taken to vanish sufficiently rapidly at the boundaries, i.e., $\epsilon(x) \rightarrow 0$, as $x \rightarrow \pm \infty$.

\section{II.2. Master Ward Identity for Diffeomorphisms}

Given the diffeomorphisms invariance of the generating functional $Z$, we may now derive a master WI associated with this symmetry. To this end, we require that the part of $Z$ containing the source terms remains invariant under the infinitesimal diffeomorphisms (II.16). To accomplish this, the sources need to transform as tensors of the relevant rank as follows:

$$
\begin{aligned}
J_{h}^{\prime \mu \nu} & =J_{h}^{\mu \nu}+\kappa\left(\epsilon^{\alpha} \partial_{\alpha} J_{h}^{\mu \nu}-J_{h}^{\nu \alpha} \partial^{\mu} \epsilon_{\alpha}-J_{h}^{\mu \alpha} \partial^{\nu} \epsilon_{\alpha}\right), \\
J_{H}^{\prime} & =J_{H}+\kappa \epsilon^{\alpha} \partial_{\alpha} J_{H} \\
J_{G}^{\prime} & =J_{G}+\kappa \epsilon^{\alpha} \partial_{\alpha} J_{G} \\
J_{\psi}^{\prime} & =J_{\psi}+\kappa \epsilon^{\alpha} \partial_{\alpha} J_{\psi} \\
\bar{J}_{\psi}^{\prime} & =\bar{J}_{\psi}+\kappa \epsilon^{\alpha} \partial_{\alpha} \bar{J}_{\psi} \\
J_{A}^{\prime \mu} & =J_{A}^{\mu}+\kappa\left(\epsilon^{\alpha} \partial_{\alpha} J_{A}^{\mu}-\epsilon_{\alpha} \partial^{\mu} J_{A}^{\alpha}\right) .
\end{aligned}
$$

Under these transformations, along with the diffeomorphisms (II.16) and (II.17), the generating functional $Z$ remains invariant. Therefore, writing $X^{\prime}=X+\delta X$ for $X \in\left\{\bar{h}_{\mu \nu}, \bar{H}, G, A_{\mu}, J_{h}^{\mu \nu}, J_{\psi}, J_{\psi}, J_{H}, J_{G}, J_{A}^{\mu}\right\}$, we obtain the identity

$$
\int d^{4} x \sum_{X} \frac{\delta Z}{\delta X} \delta X=0
$$

Defining the generating functional of connected Green's functions $W$ by

$$
Z\left[\bar{h}_{\mu \nu}, \bar{H}, \bar{G}, \bar{A}_{\mu}, J_{h}^{\mu \nu}, J_{\psi}, \bar{J}_{\psi}, J_{H}, J_{G}, J_{A}^{\mu}\right]=\exp \left(i W\left[\bar{h}_{\mu \nu}, \bar{H}, \bar{G}, \bar{A}_{\mu}, J_{h}^{\mu \nu}, J_{\psi}, \bar{J}_{\psi}, J_{H}, J_{G}, J_{A}^{\mu}\right]\right),
$$

we obtain

$$
\int d^{4} x \sum_{X} \frac{\delta W}{\delta X} \delta X=0
$$

Next, we define the one particle irreducible (1PI) effective action $\Gamma$ by means of a Legendre transform of $W$ :

$$
\begin{aligned}
\Gamma\left[\bar{h}_{\mu \nu}, \psi, \bar{\psi}, \bar{H}, \bar{G}, \bar{A}_{\mu}, h_{\mu \nu}, H, G, A_{\mu}\right]= & W\left[\bar{h}_{\mu \nu}, \bar{H}, \bar{G}, \bar{A}_{\mu}, J_{h}^{\mu \nu}, J_{\psi}, \bar{J}_{\psi}, J_{H}, J_{G}, J_{A}^{\mu}\right] \\
& -\int d^{4} x \sqrt{-\bar{g}}\left(J_{h}^{\mu \nu} h_{\mu \nu}+\bar{J}_{\psi} \psi+\bar{\psi} J_{\psi}+J_{H} H+J_{G} G+J_{A}^{\mu} A_{\mu}\right),
\end{aligned}
$$

where

$$
h_{\mu \nu} \equiv \frac{\delta W}{\delta J_{h}^{\mu \nu}}, \quad \psi \equiv \frac{\delta W}{\delta \bar{J}_{\psi}}, \quad \bar{\psi} \equiv \frac{\delta W}{\delta J_{\psi}}, \quad H \equiv \frac{\delta W}{\delta J_{H}}, \quad G \equiv \frac{\delta W}{\delta J_{G}}, \quad A_{\mu} \equiv \frac{\delta W}{\delta J_{A}^{\mu}} .
$$

To have an invariant effective action, we must require that the source terms remain invariant. As a consequence, the Legendre transform variables transform like their quantum field counterparts, i.e. according to the transformations II.16) with the identification $X^{Q} \rightarrow X$ for $X \in\left\{h_{\mu \nu}, \psi, \bar{\psi}, H, G, A_{\mu}\right\}$. This allows us to write

$$
\Gamma\left[\bar{h}_{\mu \nu}^{\prime}, \psi^{\prime}, \bar{\psi}^{\prime}, \bar{H}^{\prime}, \bar{G}^{\prime}, \bar{A}_{\mu}^{\prime}, h_{\mu \nu}^{\prime}, H^{\prime}, G^{\prime}, A_{\mu}^{\prime}\right]=\Gamma\left[\bar{h}_{\mu \nu}, \psi, \bar{\psi}, \bar{H}, \bar{G}, \bar{A}_{\mu}, h_{\mu \nu}, H, G, A_{\mu}\right] .
$$

For vanishing arguments of the quantum fields $h_{\mu \nu}, H, G, A_{\mu}$, we have

$$
\bar{\Gamma}\left[\bar{h}_{\mu \nu}^{\prime}, \psi^{\prime}, \bar{\psi}^{\prime}, \bar{H}^{\prime}, \bar{G}^{\prime}, \bar{A}_{\mu}^{\prime}\right]=\bar{\Gamma}\left[\bar{h}_{\mu \nu}, \psi, \bar{\psi}, \bar{H}, \bar{G}, \bar{A}_{\mu}\right],
$$




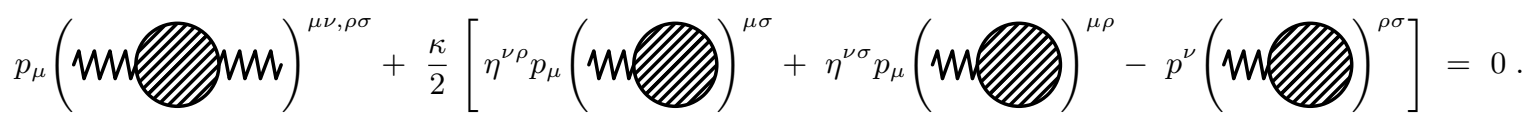

FIG. 1: Diagrammatic representation of the Ward Identity (II.34), where the zigzag lines denote gravitons.

which is a statement of invariance for the background field effective action defined by

$$
\bar{\Gamma}\left[\bar{h}_{\mu \nu}, \psi, \bar{\psi}, \bar{H}, \bar{G}, \bar{A}_{\mu}\right] \equiv \Gamma\left[\bar{h}_{\mu \nu}, \psi, \bar{\psi}, \bar{H}, \bar{G}, \bar{A}_{\mu}, 0,0,0,0\right] .
$$

An immediate consequence of this invariance is the master Ward identity

$$
\begin{aligned}
{\left[\delta_{\mu}^{\alpha} \partial_{\nu}+\kappa\left(\bar{h}_{\nu}^{\alpha} \partial_{\mu}+\partial_{\mu} \bar{h}_{\nu}^{\alpha}+\right.\right.} & \left.\left.\frac{1}{2} \partial^{\alpha} \bar{h}_{\mu \nu}\right)\right] \frac{\delta \bar{\Gamma}}{\delta \bar{h}_{\mu \nu}(x)}+\kappa\left(\partial^{\alpha} \bar{A}_{\mu}-\partial_{\mu} \bar{A}^{\alpha}-\bar{A}^{\alpha} \partial_{\mu}\right) \frac{\delta \bar{\Gamma}}{\delta \bar{A}_{\mu}} \\
& +\kappa \partial^{\alpha} \bar{H} \frac{\delta \bar{\Gamma}}{\delta \bar{H}}+\kappa \partial^{\alpha} \bar{G} \frac{\delta \bar{\Gamma}}{\delta \bar{G}}+\frac{\delta \bar{\Gamma}}{\delta \psi} \kappa \partial^{\alpha} \psi+\kappa \partial^{\alpha} \bar{\psi} \frac{\delta \bar{\Gamma}}{\delta \bar{\psi}}=0
\end{aligned}
$$

where $\alpha$ is a free index. By appropriate differentiation of $(I I .32)$ with respect to the fields of the theory, this master WI can be used to deduce further WIs and relations between correlation functions of the background fields.

Since we are interested here only in graviton correlation functions, we may take the matter field arguments of the effective action to zero. This yields a simpler version of the master WI:

$$
\left[\delta_{\mu}^{\alpha} \partial_{\nu}+\kappa\left(\bar{h}_{\nu}^{\alpha} \partial_{\mu}+\partial_{\mu} \bar{h}_{\nu}^{\alpha}+\frac{1}{2} \partial^{\alpha} \bar{h}_{\mu \nu}\right)\right] \frac{\delta \bar{\Gamma}}{\delta \bar{h}_{\mu \nu}(x)}=0
$$

Differentiating functionally with respect to $\bar{h}_{\rho \sigma}(y)$ and converting the result into the momentum space, we obtain the Ward identity

$$
p_{\mu} \Pi^{\mu \nu, \rho \sigma}(p)+\frac{\kappa}{2}\left(\eta^{\nu \rho} p_{\mu} T_{h}^{\sigma \mu}+\eta^{\nu \sigma} p_{\mu} T_{h}^{\rho \mu}-p^{\nu} T_{h}^{\rho \sigma}\right)=0
$$

where $p^{\mu}$ is the graviton momentum, $\Pi^{\mu \nu, \rho \sigma}(p)$ is the 1PI graviton self-energy and $T_{h}^{\mu \nu}$ is the 1-point correlation function for the graviton tadpoles. Figure 1 gives a graphical representation of the Ward identity (II.34), where the zigzag lines indicate gravitons.

We conclude this section by commenting on the appearance of the terms depending on the graviton tadpoles $T_{h}^{\mu \nu}$ in the Ward identity (II.34). In fact, their appearance is where Yang-Mills theory and PQG explicitly differ, as tadpole graphs for Yang-Mills fields vanish identically due to Lorentz covariance. On the other hand, previous studies in PQG mostly focused on massless particle contributions to the graviton self-energy [13 16, for which the tadpole contributions were unimportant, since these contributions vanish identically in the context of DR. Thus, the self-energy becomes transverse in this case, as a consequence of the WI (II.34), with $T_{h}^{\mu \nu}=0$. In the massive case, however, the tadpole graphs do not vanish in DR, thus signifying the presence of longitudinal modes in the graviton self-energy. In the next two sections, we will explicitly demonstrate how these longitudinal modes disappear after minimisation of the effective action and renormalization of the cosmological constant.

\section{MINIMISATION CONDITIONS AND COSMOLOGICAL CONSTANT RENORMALIZATION}

In this section, we discuss the minimization of the effective action $\Gamma$, in the presence of background graviton fields, and elucidate its connection with the renormalization of the cosmological constant $\Lambda$. We also derive a low-energy theorem that relates graviton tadpoles with the graviton self-energy at zero external momentum. As we will see, this theorem plays a central role to ensure the masslessness of gravitons.

In the context of the BFM, the minimisation of the effective action with respect to the background fields translates into the generic condition:

$$
\left.\frac{\delta \Gamma}{\delta X}\right|_{X=0}=0
$$


for $X \in\left\{h_{\mu \nu}, \psi, \bar{\psi}, H, G, A_{\mu}\right\}$. Specifically, we require that the vacuum expectation value (VEV) $v$ of the Higgs boson be translation and Lorentz invariant, i.e., $\partial_{\mu} v=0$. If we define $\Gamma=\Gamma^{(0)}+\Gamma^{(n \geq 1)}$, where $\Gamma^{(n \geq 1)}$ represents the quantum corrections, we obtain the following equations:

$$
\begin{aligned}
\frac{\delta \Gamma}{\delta H} & =f_{\bar{H}}\left(\bar{H}, \bar{G}, \bar{A}_{\mu}, v_{0}, \mu_{0}^{2}, \lambda_{0}, e_{0}\right)-\lambda_{0} v_{0}\left(v_{0}^{2}-\frac{\mu_{0}^{2}}{\lambda_{0}}\right)+\frac{\delta \Gamma^{(n \geq 1)}}{\delta H}=0 \\
\frac{\delta \Gamma}{\delta G} & =f_{\bar{G}}\left(\bar{H}, \bar{G}, \bar{A}_{\mu}, v_{0}, \mu_{0}^{2}, \lambda_{0}, e_{0}\right)+\frac{\delta \Gamma^{(n \geq 1)}}{\delta G}=0 \\
\frac{\delta \Gamma}{\delta \psi} & =\frac{\delta \Gamma^{(n \geq 1)}}{\delta \psi}=0 \\
\frac{\delta \Gamma}{\delta \bar{\psi}} & =\frac{\delta \Gamma^{(n \geq 1)}}{\delta \bar{\psi}}=0 \\
\frac{\delta \Gamma}{\delta A_{\mu}} & =f_{\bar{A}}^{\mu}\left(\bar{H}, \bar{G}, \bar{A}_{\mu}, v_{0}, \mu_{0}^{2}, \lambda_{0}, e_{0}\right)+\frac{\delta \Gamma^{(n \geq 1)}}{\delta A_{\mu}}=0 \\
\frac{\delta \Gamma}{\delta h_{\mu \nu}} & =\frac{1}{2} \bar{g}^{\mu \nu}\left(\frac{1}{\kappa} \bar{R}+\kappa\left(\Lambda_{0}+\Lambda_{0}^{H}\right)\right)-\frac{1}{\kappa} \bar{R}^{\mu \nu}-\frac{\kappa}{2} \bar{T}^{\mu \nu}+\frac{\delta \Gamma^{(n \geq 1)}}{\delta h_{\mu \nu}}=0
\end{aligned}
$$

where

$$
\begin{aligned}
f_{\bar{H}}\left(\bar{H}, \bar{G}, \bar{A}_{\mu}, v_{0}, \mu_{0}^{2}, \lambda_{0}, e_{0}\right)= & \bar{g}^{\mu \nu} \partial_{\mu} \partial_{\nu} \bar{H}+e_{0}^{2} \bar{A}^{\mu} \bar{A}_{\mu}\left(v_{0}+\bar{H}\right) \\
& -\lambda_{0}\left[v_{0}\left(2 v_{0} \bar{H}+\bar{H}^{2}+\bar{G}^{2}\right)+\bar{H}\left(\left(v_{0}+\bar{H}\right)^{2}+\bar{G}^{2}-\frac{\mu_{0}^{2}}{\lambda_{0}}\right)\right], \\
f_{\bar{G}}\left(\bar{H}, \bar{G}, \bar{A}_{\mu}, v_{0}, \mu_{0}^{2}, \lambda_{0}, e_{0}\right)= & \bar{g}^{\mu \nu} \partial_{\mu} \partial_{\nu} \bar{G}+e_{0}^{2} \bar{A}^{\mu} \bar{A}_{\mu} \bar{G}+\frac{\lambda_{0}}{2} \bar{G}\left[\left(v_{0}+\bar{H}\right)^{2}+\bar{G}^{2}-\frac{\mu_{0}^{2}}{\lambda_{0}}\right], \\
f_{\bar{A}}^{\mu}\left(\bar{H}, \bar{G}, \bar{A}_{\mu}, v_{0}, \mu_{0}^{2}, \lambda_{0}, e_{0}\right)= & \bar{g}^{\mu \rho} \bar{g}^{\nu \sigma}\left(\partial_{\nu} \partial_{\sigma} \bar{A}_{\rho}-\partial_{\rho} \partial_{\sigma} \bar{A}_{\nu}\right)-e_{0}\left(\left(v_{0}+\bar{H}\right) \partial^{\mu} \bar{G}-\bar{G} \partial^{\mu} \bar{H}\right) \\
& +e_{0}^{2} \bar{A}^{\mu}\left(\left(v_{0}+\bar{H}\right)^{2}+\bar{G}^{2}\right) \\
\Lambda_{0}^{H}= & -\frac{\lambda_{0}}{4}\left(v_{0}^{2}-\frac{\mu_{0}^{2}}{\lambda_{0}}\right)^{2}
\end{aligned}
$$

and $\bar{e}_{a}^{\mu}$ represents the background vielbein field. Here, a bar on a field (other than $\bar{\psi}$ ) represents a background field and a subscript 0 indicates a bare (unrenormalized) kinematic parameter, such as the bare coupling constant $e_{0}$ and the bare Higgs VEV $v_{0}$. In the BFM, the background fields are not free, but obey their respective equations of motion with some specified boundary conditions. Thus, we assume that all the background fields satisfy these constraints without determining their analytical form. Finally, in the present model under study, only the Higgs boson and the graviton can have non-zero tadpole contributions.

Let us now turn our attention to discussing quantum loop effects on the cosmological constant $\Lambda$. Observe that the generating functional $Z$ defined in (II.14) is well specified, except of an overall normalization constant $N$. In theories, in which gravitons are treated as classical background fields, such a constant $N$ seems to be equivalent to renormalization of $\Lambda$. However, in theories of quantum gravity, the cosmological constant is accompanied by a factor $\sqrt{-g}$, which prevents the factorization of $\Lambda$ from the path integral. To deal with this problem, we treat the cosmological term $\sqrt{-g} \Lambda$ as an interaction in the action and renormalize $\Lambda$, by renormalizing the effective action $\Gamma[0]$ by means of a gauge-invariant CT $\delta \Lambda$. This can be done by first writing $\Lambda_{0}=\Lambda+\delta \Lambda$ and then imposing the condition

$$
\Gamma[0]=\Lambda_{0}+\Lambda_{0}^{H}+\Gamma^{(n \geq 1)}[0]=\Lambda
$$

Assuming a flat Minkowski background after renormalization, we set $\Lambda=0$, such that $\eta_{\mu \nu}$ remains a solution of the background equations of motion. Notice that $\Lambda_{0}^{H}$ is renormalized only through the Higgs VEV $v_{0}$ and the quartic coupling constant $\lambda_{0}$ [cf. (III.13)]. At the one-loop level, the contribution $\Gamma^{(1)}[0]$ to the cosmological constant may 
graphically be represented as

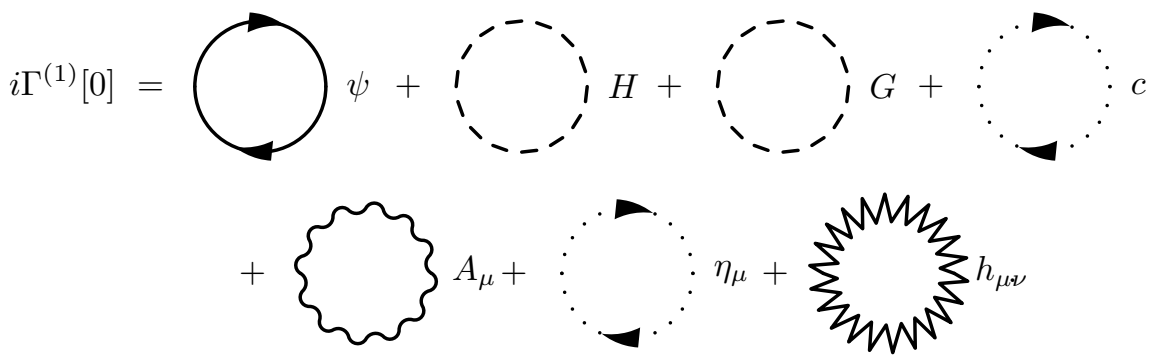

Writing $\Lambda_{0}^{H}=\Lambda_{H}+\delta \Lambda_{H}$, it is not difficult to see that $\delta \Lambda_{H}=0$ at the one-loop level. Therefore, the renormalization condition (III.14) simplifies to

$$
\delta \Lambda+\Gamma^{(1)}[0]=0 .
$$

In the DR scheme, the individual graphs contributing to $\Gamma^{(1)}[0]$ can be calculated explicitly. In this way, we obtain

$$
\begin{aligned}
& \vdots \begin{array}{l}
y_{1} \\
\vdots
\end{array}
\end{aligned}
$$

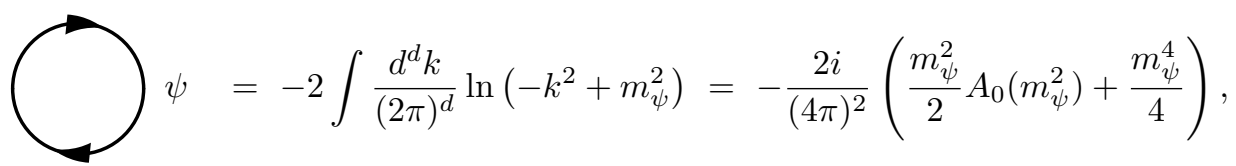

$$
\begin{aligned}
& \left\{A_{\mu}=\frac{d-1}{2} \int \frac{d^{d} k}{(2 \pi)^{d}} \ln \left(-k^{2}+m_{A}^{2}\right)+\frac{1}{2} \int \frac{d^{d} k}{(2 \pi)^{d}} \ln \left(-k^{2}+\xi_{G} m_{A}^{2}\right)\right. \\
& =\frac{3 i}{2(4 \pi)^{2}}\left(\frac{m_{A}^{2}}{2} A_{0}\left(m_{A}^{2}\right)-\frac{m_{A}^{4}}{12}\right)+\frac{i}{2(4 \pi)^{2}}\left(\frac{\xi_{G} m_{A}^{2}}{2} A_{0}\left(\xi_{G} m_{A}^{2}\right)+\frac{\xi_{G}^{2} m_{A}^{4}}{4}\right), \\
& \vdots \begin{array}{l}
\vdots \\
\vdots
\end{array} \\
& \therefore \quad \vdots c=-\int \frac{d^{d} k}{(2 \pi)^{d}} \ln \left(-k^{2}+\xi_{G} m_{A}^{2}\right)=-\frac{i}{(4 \pi)^{2}}\left(\frac{\xi_{G} m_{A}^{2}}{2} A_{0}\left(\xi_{G} m_{A}^{2}\right)+\frac{\xi_{G}^{2} m_{A}^{4}}{4}\right) \text {, } \\
& \therefore \quad \vdots \eta_{\mu}=0, \\
& \sum_{W W}^{N M L^{2}} \xi_{h \nu}=0 .
\end{aligned}
$$

Here, $A_{0}\left(m^{2}\right)$ is the tadpole loop integral defined in $d=4-2 \epsilon$ as

$$
A_{0}\left(m^{2}\right) \equiv(2 \pi \mu)^{4-d} \int \frac{d^{d} k}{i \pi^{2}} \frac{1}{k^{2}-m^{2}}=m^{2}\left[\frac{1}{\bar{\epsilon}}+1-\ln \left(\frac{m^{2}}{\mu^{2}}\right)\right]
$$

where $1 / \bar{\epsilon}=1 / \epsilon-\gamma_{E}+\ln 4 \pi$, with $\gamma_{E}$ being the Euler-Mascheroni constant and $\mu$ the 't Hooft mass renormalization scale. We note that the sum

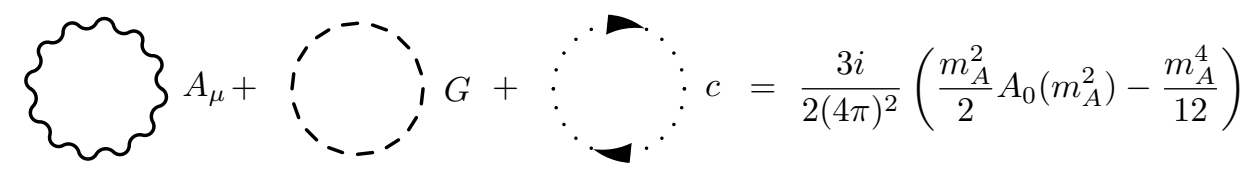


is independent of the $U(1)_{Y}$ gauge fixing parameter $\xi_{G}$. Thus, at the one-loop level, the cosmological constant CT $\delta \Lambda$ is found to be

$$
\delta \Lambda=\frac{2}{(4 \pi)^{2}}\left(\frac{m_{\psi}^{2}}{2} A_{0}\left(m_{\psi}^{2}\right)+\frac{m_{\psi}^{4}}{4}\right)-\frac{1}{2(4 \pi)^{2}}\left(\frac{m_{H}^{2}}{2} A_{0}\left(m_{H}^{2}\right)+\frac{m_{H}^{4}}{4}\right)-\frac{3}{2(4 \pi)^{2}}\left(\frac{m_{A}^{2}}{2} A_{0}\left(m_{A}^{2}\right)-\frac{m_{A}^{4}}{12}\right) .
$$

The fact that $\delta \Lambda$ is independent of $\xi_{G}$ and the diffeomorphisms-fixing parameters $\xi_{D}$ and $\sigma$ reflects the gauge invariance of the effective action at its minimum [46] and provides a consistency check for the correctness of our analytic results.

Let us now analyze the minimisation conditions (III.2) and (III.7) related to the Higgs and the graviton tadpoles, respectively. For the Higgs tadpole condition, we have

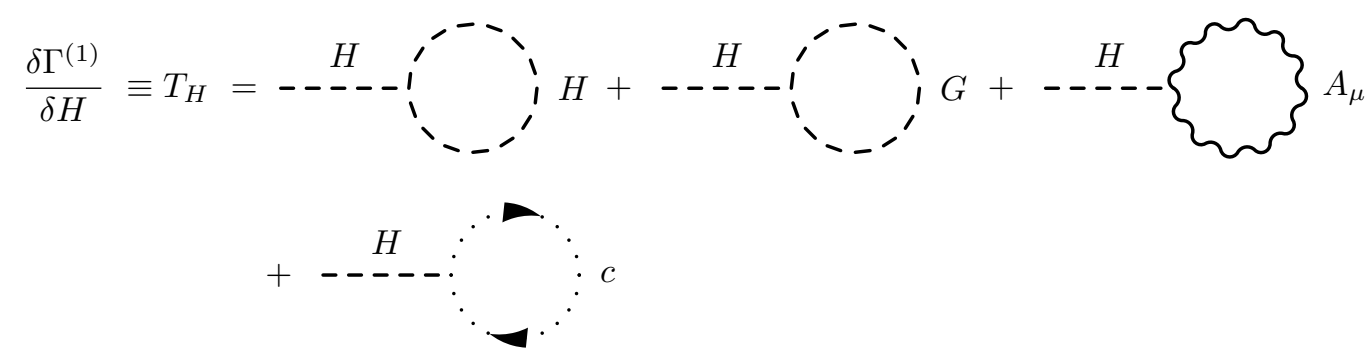

Expressing the bare Higgs VEV $v_{0}$ as $v_{0}=v+\delta v$, III.2 reads

$$
-2 \lambda v^{2} \delta v+T_{H}=0
$$

at the one-loop level, from which we deduce the Higgs VEV CT

$$
\delta v=\frac{T_{H}}{2 \lambda v^{2}} .
$$

To deal with the graviton tadpole condition (III.7), we first define

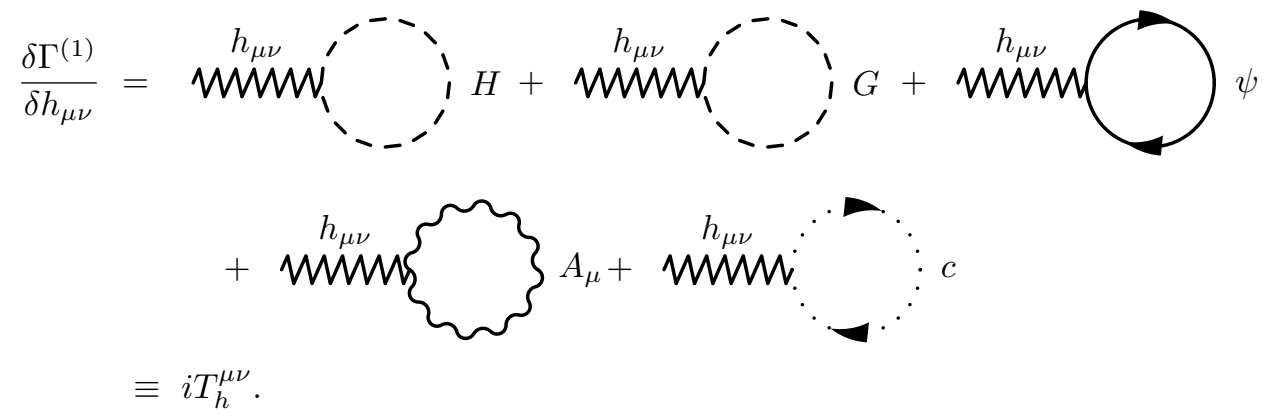

As done with the Higgs field, we allow for the quantum graviton field $h_{\mu \nu}$ to develop a VEV, by replacing $h_{\mu \nu} \rightarrow$ $h_{\mu \nu}+\delta h_{\mu \nu}$. In this way, the one-loop minimization condition for the graviton field becomes:

$$
\int d^{4} y\left[\left.\frac{\delta^{2} S}{\delta h_{\mu \nu}(x) \delta h_{\rho \sigma}(y)}\right|_{g_{\mu \nu}=\eta_{\mu \nu}} \delta h_{\rho \sigma}(y)\right] z+\frac{\kappa}{2} \eta^{\mu \nu} \delta \Lambda+T_{h}^{\mu \nu}=0 .
$$

From this last equation, we easily see that the second functional derivative with respect to the quantum graviton field is the inverse graviton propagator in the flat space, i.e.

$$
\left.\frac{\delta^{2} S}{\delta h_{\mu \nu}(x) \delta h_{\rho \sigma}(y)}\right|_{g_{\mu \nu}=\eta_{\mu \nu}}=\Delta^{-1 \mu \nu \rho \sigma}(x-y) .
$$

By virtue of the latter, III.31 may be recast into the form:

$$
\int d^{4} y\left[\Delta^{-1 \mu \nu \rho \sigma}(x-y) \delta h_{\rho \sigma}(y)\right]+\frac{\kappa}{2} \eta^{\mu \nu} \delta \Lambda+T_{h}^{\mu \nu}(x)=0 .
$$


Solving equation III.33) for $\delta h_{\rho \sigma}$ yields

$$
\delta h_{\rho \sigma}(x)=-\int d^{4} y \Delta_{\mu \nu \rho \sigma}(x-y)\left(T_{h}^{\rho \sigma}(y)+\frac{\kappa}{2} \eta^{\rho \sigma} \delta \Lambda\right) .
$$

It is now instructive to calculate the one-loop graviton tadpole $T_{h}^{\mu \nu}$ resulting from our Abelian Higgs model. With the aid of the Feynman rules given in Appendix A, the individual contributions to $T_{h}^{\mu \nu}$ are given by

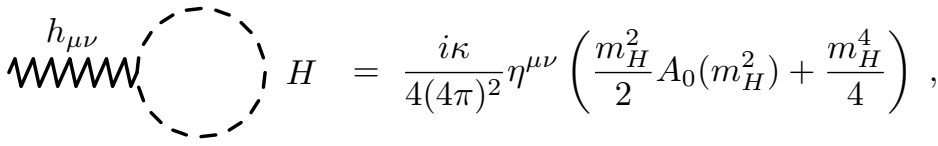

$$
\begin{aligned}
& \mathrm{WWWW}_{\mu \nu}^{h_{\mu \nu}}, G=\frac{i \kappa}{4(4 \pi)^{2}} \eta^{\mu \nu}\left(\frac{\xi_{G} m_{A}^{2}}{2} A_{0}\left(\xi_{G} m_{A}^{2}\right)+\frac{\xi_{G}^{2} m_{A}^{4}}{4}\right) \text {, } \\
& \operatorname{WMM}\left(\longrightarrow=-\frac{i \kappa}{2(4 \pi)^{2}} \eta^{\mu \nu}\left(\frac{m_{\psi}^{2}}{2} A_{0}\left(m_{\psi}^{2}\right)+\frac{m_{\psi}^{4}}{4}\right),\right. \\
& \left.\operatorname{WMU}_{\mu \nu}^{h_{\mu \nu}}\right\} A_{\mu}=\frac{3 i \kappa}{4(4 \pi)^{2}} \eta^{\mu \nu}\left(\frac{m_{A}^{2}}{2} A_{0}\left(m_{A}^{2}\right)-\frac{m_{A}^{4}}{12}\right) \\
& +\frac{i \kappa}{4(4 \pi)^{2}} \eta^{\mu \nu}\left(\frac{\xi_{G} m_{A}^{2}}{2} A_{0}\left(\xi_{G} m_{A}^{2}\right)-\frac{\xi_{G}^{2} m_{A}^{4}}{12}\right), \\
& \text { WWMU } \quad \vdots c=-\frac{i \kappa}{(4 \pi)^{2}} \eta^{\mu \nu}\left(\frac{\xi_{G} m_{A}^{2}}{2} A_{0}\left(\xi_{G} m_{A}^{2}\right)+\frac{\xi_{G}^{2} m_{A}^{4}}{4}\right) \text {. }
\end{aligned}
$$

Interestingly enough, we observe that the sum

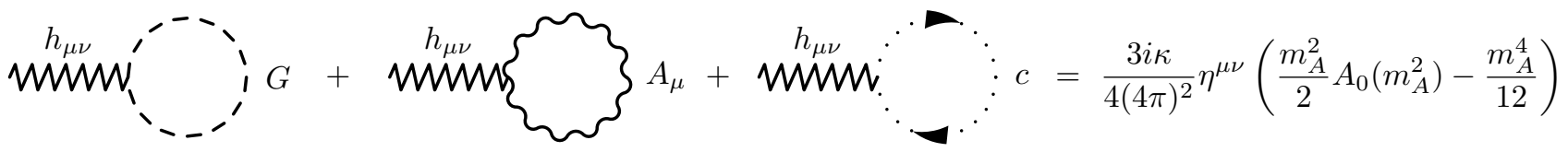

is independent of the gauge fixing parameter $\xi_{G}$, implying that the graviton tadpoles form a gauge-invariant set of graphs. This is in stark contrast with the Higgs tadpole and its VEV CT $\delta v$, which are known to be both gaugedependent quantities (e.g., see [45]).

Our effort to gain a better understanding of the gauge-fixing parameter independence of $T_{h}^{\mu \nu}$ led us to observe the following relation:

$$
T_{h}^{\mu \nu}+\frac{\kappa}{2} \eta^{\mu \nu} \delta \Lambda=0 .
$$

Remarkably, III.41 holds separately for each of the quantum field circulating in the loop. Hence, at the one-loop level, tadpole graphs are directly linked with the gauge-invariant renormalization CT $\delta \Lambda$ of the cosmological constant, so $T_{h}^{\mu \nu}$ is a gauge-invariant quantity as well. Moreover, graviton tadpole graphs cancel against the CT $\delta \Lambda$ in the one-loop effective action, which implies that there is no VEV renormalization for the graviton field, i.e. $\delta h_{\mu \nu}=0$.

It is important to stress here that our approach to renormalizing the graviton field differs significantly from the one outlined, e.g., in [12, where a cosmological constant was introduced in an ad hoc manner, in order to cancel the graviton tadpole effects. In our case, such a cancellation is a result of an explicit computation, without the need to impose an additional constraint. In the next subsection, we will show that the relation (III.41) leading to the non-renormalization of the graviton VEV, with $\delta h_{\mu \nu}=0$, is not an one-loop coincidence, but a result that holds to all orders in perturbation for a gravitational theory renormalized to a Minkowski flat background. 


\section{III.1. The Graviton Low Energy Theorem}

Here we will explicitly demonstrate how the relation (III.41) holds true to all orders in perturbation. As we will see, this non-perturbative relation is a direct consequence of a Graviton Low Energy Theorem (GLET).

Given the conceptual similarity of the GLET with the so-called Higgs-boson Low Energy Theorem (HLET) 40 45], we begin our demonstration by briefly reminding the reader of the latter. The HLET may be stated by the following defining equation:

$$
\frac{\partial}{\partial v} \Gamma=\frac{\delta \Gamma}{\delta \bar{H}(0)},
$$

where $\bar{H}(0)$ denotes a zero-momentum background Higgs field. This result may be derived from a global shift symmetry that exists between the Higgs VEV $v$ and the background Higgs field $\bar{H}$ of the form:

$$
v^{\prime}=v+s, \quad \bar{H}^{\prime}=\bar{H}-s,
$$

for some infinitesimal constant $s$, provided a compatible gauge-fixing condition is chosen [45. Taking a functional derivative with respect to $\bar{H}$, invoking momentum conservation and writing $\Gamma=\Gamma^{(0)}+\Gamma^{(n \geq 1)}$, where $\Gamma^{(n \geq 1)}$ represents the part of the action containing one- and higher-order quantum loop effects, we obtain

$$
\frac{\partial}{\partial v}\left(\frac{\delta \Gamma^{(n \geq 1)}}{\delta \bar{H}(0)}\right)=\frac{\delta^{2} \Gamma^{(n \geq 1)}}{\delta \bar{H}(0) \delta \bar{H}(0)} .
$$

Therefore, one consequence of the HLET relevant to our discussion here is the relation of the Higgs-boson tadpole to quantum effects on the Higgs-boson mass.

We may now try to extend the basic idea of HLET to theories of quantum gravity. As discussed in Section II the full spacetime metric $g_{\mu \nu}$ may be decomposed in the BFM framework of quantum gravity as follows:

$$
g_{\mu \nu}=\eta_{\mu \nu}+\kappa\left(\bar{h}_{\mu \nu}+h_{\mu \nu}\right) \text {, }
$$

where $\bar{g}_{\mu \nu}=\eta_{\mu \nu}+\kappa \bar{h}_{\mu \nu}$ is the background metric [cf. [II.8]]. In close analogy to HLET, it is not difficult to observe that there is a similar symmetry for the effective action $\Gamma$ of the complete matter-gravity theory. In particular, the effective action $\Gamma$ remains invariant under the shift transformations:

$$
\eta_{\mu \nu}^{\prime}=\eta_{\mu \nu}+s_{\mu \nu}, \quad \bar{h}_{\mu \nu}^{\prime}=\bar{h}_{\mu \nu}-\frac{1}{\kappa} s_{\mu \nu}
$$

where $s_{\mu \nu}$ is an arbitrary tensor. Since the generating functional (II.14) remains invariant under the shift symmetry (III.46), we can derive the shift Ward identity:

$$
\frac{\partial Z}{\partial \eta_{\mu \nu}}-\frac{1}{\kappa} \int d^{4} x \frac{\delta Z}{\delta \bar{h}_{\mu \nu}(x)}=0
$$

which implies

$$
\frac{\partial W}{\partial \eta_{\mu \nu}}-\frac{1}{\kappa} \int d^{4} x \frac{\delta W}{\delta \bar{h}_{\mu \nu}(x)}=0
$$

by virtue of (II.25). With the aid of (II.27), we may translate the last result into the shift WI for the effective action:

$$
\frac{\partial \Gamma}{\partial \eta_{\mu \nu}}-\frac{1}{\kappa} \int d^{4} x \frac{\delta \Gamma}{\delta \bar{h}_{\mu \nu}(x)}=0
$$

or equivalently in momentum space:

$$
\kappa \frac{\partial}{\partial \eta_{\mu \nu}} \Gamma=\frac{\delta \Gamma}{\delta \bar{h}_{\mu \nu}(0)} .
$$

Equation III.50 is the defining equation for the GLET, where $\bar{h}_{\mu \nu}(0)$ is a zero-momentum graviton field. Now, if we consider the counterterm in the effective action,

$$
\Delta S=\int d^{4} x \sqrt{-g} \delta \Lambda
$$


in order to cancel $\Gamma^{(n \geq 1)}[0]$, we obtain the relation III.41):

$$
T_{h}^{\mu \nu}+\frac{\kappa}{2} \eta^{\mu \nu} \delta \Lambda=0,
$$

which holds true to all orders in perturbation theory. Hence, the one-loop relation (III.41) is a consequence of the GLET.

In addition to relating the graviton tadpole to the cosmological constant, the GLET can also relate the graviton tadpole to the graviton self-energy at zero external momentum:

$$
\kappa \frac{\partial}{\partial \eta_{\mu \nu}}\left(\frac{\delta \Gamma^{(n \geq 1)}}{\delta \bar{h}_{\rho \sigma}(0)}\right)=\frac{\delta^{2} \Gamma^{(n \geq 1)}}{\delta \bar{h}_{\mu \nu}(0) \delta \bar{h}_{\rho \sigma}(0)} .
$$

Since graviton tadpoles vanish identically for massless fields in the loop in the DR scheme, the graviton self-energy at zero external momentum will vanish as well, by means of (III.52). Consequently, the GLET (III.50) can also guarantee the masslessness of the graviton field in DR, if all particles in the quantum loops are massless. As we will see in the next section, however, this is not in general true, if massive particles occur in the graviton self-energy. In this case, both the GLET (III.50) and the diffeomorphisms WI (II.34) will be needed to render the graviton massless to all orders in perturbation, assuming a flat Minkowski background.

\section{MATTER CONTRIBUTIONS TO THE GRAVITON SELF-ENERGY}

In this section, we will first demonstrate explicitly how upon renormalization, the graviton self-energy obeys the property of transversality entailing in a massless graviton field. Subsequently, we will compute the matter contributions to the graviton self-energy tensor resulting from massive scalar, pseudo-scalar, fermion and vector-boson particles in the loops.

\section{IV.1. Transversality of the Graviton Self-energy}

The graviton self-energy transition $\bar{h}_{\mu \nu}(p) \rightarrow \bar{h}_{\rho \sigma}(p)$, which we denote as $\Pi^{\mu \nu, \rho \sigma}(p)$, receives two renormalizations: (i) from the bare cosmological constant $\Lambda_{0}$ which induces a CT proportional to $\delta \Lambda$ for the graviton mass in the effective action [cf. [III.51]]; (ii) from the Ricci scalar $R$ and the higher-dimensional operators $R^{2}$ and $R^{\mu \nu} R_{\mu \nu}$. The latter contributions (ii), which we denote as $\Delta \Pi^{\mu \nu, \rho \sigma}(p)$, are transverse in the minimal subtraction scheme ( $\left.\overline{\mathrm{MS}}\right)$ of renormalization and they have therefore no effect on the graviton mass.

Taking into account the two contributions mentioned above, the renormalized graviton self-energy $\Pi_{\mathrm{R}}^{\mu \nu, \rho \sigma}(p)$ may then be written down as follows:

$$
\Pi_{\mathrm{R}}^{\mu \nu, \rho \sigma}(p)=\Pi^{\mu \nu, \rho \sigma}(p)-\frac{\kappa^{2}}{4} P^{\mu \nu \rho \sigma} \delta \Lambda+\Delta \Pi^{\mu \nu, \rho \sigma}(p) .
$$

where we have defined the tensor

$$
P^{\mu \nu \rho \sigma} \equiv \eta^{\mu \rho} \eta^{\nu \sigma}+\eta^{\mu \rho} \eta^{\nu \sigma}-\eta^{\mu \nu} \eta^{\rho \sigma}
$$

for brevity. Employing the identity (III.41) deduced from the GLET, we may readily obtain the relation

$$
\frac{\kappa}{2} P^{\mu \nu \rho \sigma} \delta \Lambda=-\eta^{\nu \rho} T_{h}^{\sigma \mu}-\eta^{\nu \sigma} T_{h}^{\rho \mu}+\eta^{\mu \nu} T_{h}^{\rho \sigma} .
$$

Substituting this last expression back in (IV.1) gives

$$
\Pi_{\mathrm{R}}^{\mu \nu, \rho \sigma}(p)=\Pi^{\mu \nu, \rho \sigma}(p)+\frac{\kappa}{2}\left(\eta^{\nu \rho} T_{h}^{\sigma \mu}+\eta^{\nu \sigma} T_{h}^{\rho \mu}-\eta^{\mu \nu} T_{h}^{\rho \sigma}\right)+\Delta \Pi^{\mu \nu, \rho \sigma}(p) .
$$

Based on the WI (II.34) of diffeomorphisms depicted graphically in Fig. 1 and the fact that $p_{\mu} \Delta \Pi^{\mu \nu, \rho \sigma}(p)=0$, it is not difficult to show that the renormalized graviton self-energy is transverse, i.e.

$$
p_{\mu} \Pi_{\mathrm{R}}^{\mu \nu, \rho \sigma}(p)=0 .
$$

Hence, the longitudinal modes of the graviton self-energy are successfully removed after renormalizing the cosmological constant. We shall use the transversality identity (IV.5) to check the consistency of our analytic results. 


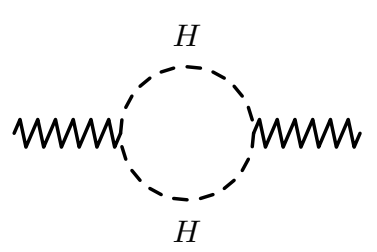

(a)

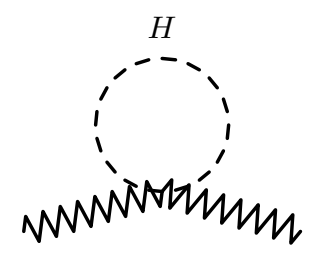

(b)

FIG. 2: The Higgs contribution to the graviton self-energy.

We may now decompose the renormalized graviton self-energy tensor $\Pi_{\mathrm{R}}^{\mu \nu, \rho \sigma}(p)$ in terms of independent rank-4 Lorentz tensors that depend on $\eta^{\mu \nu}$ and $p^{\mu} p^{\nu}$. More explicitly, $\Pi_{\mathrm{R}}^{\mu \nu, \rho \sigma}(p)$ may be expressed as follows:

$$
\begin{aligned}
\Pi_{\mathrm{R}}^{\mu \nu, \rho \sigma}(p)= & p^{\mu} p^{\nu} p^{\rho} p^{\sigma} F_{1}\left(p^{2}\right)+\eta^{\mu \nu} \eta^{\rho \sigma} F_{2}\left(p^{2}\right)+\left(\eta^{\mu \rho} \eta^{\nu \sigma}+\eta^{\nu \rho} \eta^{\mu \sigma}\right) F_{3}\left(p^{2}\right) \\
& +\left(\eta^{\mu \nu} p^{\rho} p^{\sigma}+\eta^{\rho \sigma} p^{\mu} p^{\nu}\right) F_{4}\left(p^{2}\right)+\left(\eta^{\mu \rho} p^{\nu} p^{\sigma}+\eta^{\nu \rho} p^{\mu} p^{\sigma}+\eta^{\mu \sigma} p^{\nu} p^{\rho}+\eta^{\nu \sigma} p^{\mu} p^{\rho}\right) F_{5}\left(p^{2}\right)
\end{aligned}
$$

where $F_{i}$ (with $i=1,2, \ldots, 5$ ) is a set of form factors. Note that the form factors $F_{i}$ are not independent of each other, as they have to satisfy the transversality condition (IV.5), which gives rise to following set of relations:

$$
\begin{aligned}
p^{2} F_{1}+F_{4}+2 F_{5} & =0, \\
F_{2}+p^{2} F_{4} & =0, \\
F_{3}+p^{2} F_{5} & =0 .
\end{aligned}
$$

Finally, it is important to remark here that the UV-infinite contributions of $\Delta \Pi^{\mu \nu, \rho \sigma}(p)$ to the form factors $F_{i}$ satisfy independently the three relations given in (IV.7).

\section{IV.2. Massive Scalar Loops}

First, we consider the Higgs-scalar effects on the graviton self-energy, as described by the two diagrams (a) and (b) in Fig. 2. These are given by the loop integrals

$$
\begin{aligned}
& i \Pi_{2(a)}^{\mu \nu, \rho \sigma}(p)=\frac{1}{2} \int \frac{d^{d} k}{(2 \pi)^{d}} V_{H H h}^{\mu \nu}\left(k,-(p+k), m_{H}\right) V_{H H h}^{\rho \sigma}\left(-k, p+k, m_{H}\right)\left[\frac{i}{k^{2}-m_{H}^{2}}\right]\left[\frac{i}{(p+k)^{2}-m_{H}^{2}}\right], \\
& i \Pi_{2(b)}^{\mu \nu, \rho \sigma}(p)=\frac{1}{2} \int \frac{d^{d} k}{(2 \pi)^{d}} V_{H H h h}^{\mu \nu \rho \sigma}\left(k,-k, m_{H}\right)\left[\frac{i}{k^{2}-m_{H}^{2}}\right] .
\end{aligned}
$$

Note that the contribution of the would-be Goldstone boson $G$ is obtained by replacing $m_{H}^{2} \rightarrow \xi_{G} m_{A}^{2}$ in the above two expressions.

Without including the CTs contained in $\Delta \Pi^{\mu \nu, \rho \sigma}(p)$, the Higgs contributions to the form factors are given by

$$
\begin{aligned}
& F_{1}\left(p^{2}\right)=\frac{\kappa^{2}}{3600(4 \pi)^{2}\left(p^{2}\right)^{2}}\left[\left(\alpha_{1}+\alpha_{4}\right) B_{0}\left(p^{2}, m_{H}^{2}, m_{H}^{2}\right)+\left(\alpha_{2}+\alpha_{5}\right) A_{0}\left(m_{H}^{2}\right)+\left(\alpha_{3}+\alpha_{6}\right)\right] \\
& F_{2}\left(p^{2}\right)=\frac{\kappa^{2}}{3600(4 \pi)^{2}}\left(\alpha_{1} B_{0}\left(p^{2}, m_{H}^{2}, m_{H}^{2}\right)+\alpha_{2} A_{0}\left(m_{H}^{2}\right)+\alpha_{3}\right) \\
& F_{3}\left(p^{2}\right)=\frac{\kappa^{2}}{7200(4 \pi)^{2}}\left(\alpha_{4} B_{0}\left(p^{2}, m_{H}^{2}, m_{H}^{2}\right)+\alpha_{5} A_{0}\left(m_{H}^{2}\right)+\alpha_{6}\right) \\
& F_{4}\left(p^{2}\right)=-\frac{\kappa^{2}}{3600(4 \pi)^{2} p^{2}}\left(\alpha_{1} B_{0}\left(p^{2}, m_{H}^{2}, m_{H}^{2}\right)+\alpha_{2} A_{0}\left(m_{H}^{2}\right)+\alpha_{3}\right) \\
& F_{5}\left(p^{2}\right)=-\frac{\kappa^{2}}{7200(4 \pi)^{2} p^{2}}\left(\alpha_{4} B_{0}\left(p^{2}, m_{H}^{2}, m_{H}^{2}\right)+\alpha_{5} A_{0}\left(m_{H}^{2}\right)+\alpha_{6}\right)
\end{aligned}
$$


where

$$
\begin{aligned}
& \alpha_{1}=15\left[8 m_{H}^{4}+16 m_{H}^{2} p^{2}+3\left(p^{2}\right)^{2}\right], \\
& \alpha_{2}=-30\left(4 m_{H}^{2}+3 p^{2}\right), \\
& \alpha_{3}=120 m_{H}^{4}+220 m_{H}^{2} p^{2}-42\left(p^{2}\right)^{2}, \\
& \alpha_{4}=15\left(p^{2}-4 m_{H}^{2}\right)^{2}, \\
& \alpha_{5}=-30\left(8 m_{H}^{2}+p^{2}\right), \\
& \alpha_{6}=16\left[15 m_{H}^{4}-10 m_{H}^{2} p^{2}+\left(p^{2}\right)^{2}\right] .
\end{aligned}
$$

In addition, $B_{0}$ is the one-loop scalar self-energy integral defined in $d=4-2 \epsilon$ as

$$
\begin{aligned}
B_{0}\left(p^{2}, m_{1}^{2}, m_{2}^{2}\right) \equiv & (2 \pi \mu)^{4-d} \int \frac{d^{d} k}{i \pi^{2}} \frac{1}{k^{2}-m_{1}^{2}} \frac{1}{(k+p)^{2}-m_{2}^{2}}=\frac{1}{\bar{\epsilon}}+2-\ln \left(\frac{m_{1} m_{2}}{\mu^{2}}\right) \\
& +\frac{1}{p^{2}}\left[\left(m_{2}^{2}-m_{1}^{2}\right) \ln \left(\frac{m_{1}}{m_{2}}\right)+\lambda^{1 / 2}\left(p^{2}, m_{1}^{2}, m_{2}^{2}\right) \cosh ^{-1}\left(\frac{m_{1}^{2}+m_{2}^{2}-p^{2}}{2 m_{1} m_{2}}\right)\right],
\end{aligned}
$$

with $\lambda(x, y, z) \equiv(x-y-z)^{2}-4 y z$. For $p^{2}=0$ and $m_{1}=m_{2}=m$, the loop function $B_{0}\left(0, m^{2}, m^{2}\right)$ is related to the tadpole loop integral $A_{0}\left(m^{2}\right)$ as follows:

$$
A_{0}\left(m^{2}\right)=m^{2}\left(1+B_{0}\left(0, m^{2}, m^{2}\right)\right)
$$

We may now calculate the CTs described by $\Delta \Pi^{\mu \nu, \rho \sigma}(p)$ in the $\overline{\mathrm{MS}}$ scheme of renormalization [47. For the Higgs and Goldstone effects, these CTs may be represented by the following set of diffeomorphisms invariant operators:

$$
\begin{aligned}
\Delta S_{H} & =-\int d^{4} x \frac{\sqrt{-g}}{2(4 \pi)^{2} \bar{\epsilon}}\left[\frac{m_{H}^{2}}{6} R+\frac{1}{120} R^{2}+\frac{1}{60} R^{\mu \nu} R_{\mu \nu}\right], \\
\Delta S_{G} & =-\int d^{4} x \frac{\sqrt{-g}}{2(4 \pi)^{2} \bar{\epsilon}}\left[\frac{\xi_{G} m_{A}^{2}}{6} R+\frac{1}{120} R^{2}+\frac{1}{60} R^{\mu \nu} R_{\mu \nu}\right] .
\end{aligned}
$$

We note that the CTs in $\Delta S_{H}$ agree with [12, after making the obvious replacement: $1 / \epsilon \rightarrow 1 / \bar{\epsilon}$. The inclusion of the CTs given by $\Delta S_{H}$ and $\Delta S_{G}$ has the effect to remove simply the $1 / \bar{\epsilon}$ poles that occur through the loop integrals $A_{0}$ and $B_{0}$ in the form factors $F_{1,2, \ldots .5}$ listed in (IV.10). Finally, observe that the five form factors $F_{1,2, \ldots, 5}$ satisfy the transversality relations given in (IV.7).

\section{IV.3. Massive Fermion Loops}

Quantum loops due to a Dirac fermion $\psi$ contribute also to the graviton self-energy by the two diagrams (a) and (b) shown in Fig. 3. These two diagrams may be calculated by

$$
\begin{aligned}
& i \Pi_{3(a)}^{\mu \nu, \rho \sigma}(p)=-\int \frac{d^{d} k}{(2 \pi)^{d}} \operatorname{Tr}\left(V_{\bar{\psi} \psi h}^{\mu \nu}\left(k,-(p+k), m_{\psi}\right)\left[\frac{i(\not k+m)}{k^{2}-m_{\psi}^{2}}\right] V_{\bar{\psi} \psi h}^{\rho \sigma}\left(-k, p+k, m_{\psi}\right)\left[\frac{i(\not p+\not k+m)}{(p+k)^{2}-m_{\psi}^{2}}\right]\right), \\
& i \Pi_{3(b)}^{\mu \nu, \rho \sigma}(p)=-\int \frac{d^{d} k}{(2 \pi)^{d}} \operatorname{Tr}\left(V_{\bar{\psi} \psi h h}^{\mu \nu \rho \sigma}\left(k,-k, m_{H}\right)\left[\frac{i(\not k+m)}{k^{2}-m_{\psi}^{2}}\right]\right) .
\end{aligned}
$$

Upon including only the cosmological constant $\mathrm{CT} \delta \Lambda$, we arrive at the following analytic expressions for the form 


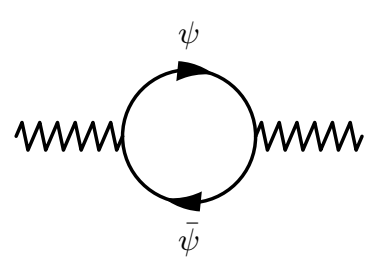

(a)

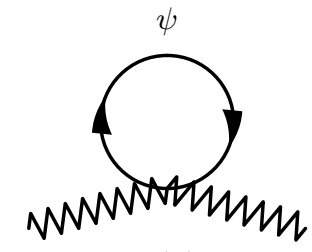

(b)

FIG. 3: The fermion contribution to the graviton self-energy.

factors:

$$
\begin{aligned}
& F_{1}\left(p^{2}\right)=\frac{\kappa^{2}}{1800(4 \pi)^{2}\left(p^{2}\right)^{2}}\left[\left(\alpha_{1}+\alpha_{4}\right) B_{0}\left(p^{2}, m_{H}^{2}, m_{H}^{2}\right)+\left(\alpha_{2}+\alpha_{5}\right) A_{0}\left(m_{H}^{2}\right)+\left(\alpha_{3}+\alpha_{6}\right)\right] \\
& F_{2}\left(p^{2}\right)=\frac{\kappa^{2}}{1800(4 \pi)^{2}}\left(\alpha_{1} B_{0}\left(p^{2}, m_{\psi}^{2}, m_{\psi}^{2}\right)+\alpha_{2} A_{0}\left(m_{\psi}^{2}\right)+\alpha_{3}\right) \\
& F_{3}\left(p^{2}\right)=\frac{\kappa^{2}}{3600(4 \pi)^{2}}\left(\alpha_{4} B_{0}\left(p^{2}, m_{\psi}^{2}, m_{\psi}^{2}\right)+\alpha_{5} A_{0}\left(m_{\psi}^{2}\right)+\alpha_{6}\right), \\
& F_{4}\left(p^{2}\right)=-\frac{\kappa^{2}}{1800(4 \pi)^{2} p^{2}}\left(\alpha_{1} B_{0}\left(p^{2}, m_{\psi}^{2}, m_{\psi}^{2}\right)+\alpha_{2} A_{0}\left(m_{\psi}^{2}\right)+\alpha_{3}\right) \\
& F_{5}\left(p^{2}\right)=-\frac{\kappa^{2}}{3600(4 \pi)^{2} p^{2}}\left(\alpha_{4} B_{0}\left(p^{2}, m_{\psi}^{2}, m_{\psi}^{2}\right)+\alpha_{5} A_{0}\left(m_{\psi}^{2}\right)+\alpha_{6}\right),
\end{aligned}
$$

with

$$
\begin{aligned}
& \alpha_{1}=-15\left(p^{2}-4 m_{\psi}^{2}\right)^{2} \\
& \alpha_{2}=30\left(8 m_{\psi}^{2}+p^{2}\right) \\
& \alpha_{3}=-16\left[15 m_{\psi}^{4}-10 m_{\psi}^{2} p^{2}+\left(p^{2}\right)^{2}\right] \\
& \alpha_{4}=-15\left[32 m_{\psi}^{4}+4 m_{\psi}^{2} p^{2}-3\left(p^{2}\right)^{2}\right] \\
& \alpha_{5}=-30\left(3 p^{2}-16 m_{\psi}^{2}\right) \\
& \alpha_{6}=-480 m_{\psi}^{4}+20 m_{\psi}^{2} p^{2}+18\left(p^{2}\right)^{2} .
\end{aligned}
$$

The UV poles proportional to $1 / \bar{\epsilon}$ that enter the form factors $F_{1,2, \ldots, 5}$ through the loop integrals $A_{0}$ and $B_{0}$ may be renormalized after taking into consideration the $\mathrm{CT}$ effective action

$$
\Delta S_{\psi}=\int d^{4} x \frac{\sqrt{-g}}{(4 \pi)^{2} \bar{\epsilon}}\left[\frac{m_{\psi}^{2}}{6} R-\frac{1}{60} R^{2}+\frac{1}{20} R^{\mu \nu} R_{\mu \nu}\right]
$$

This last result is in agreement with [48]. As with the scalar case, it is not difficult to check that the form factors $F_{1,2, \ldots, 5}$ exhibited in IV.18 satisfy the transversality relations in IV.7).

\section{IV.4. Massive Gauge and Ghost Loops}

Finally, we consider quantum loop effects of a massive gauge boson $A_{\mu}$ and their respective ghost fields $c$ and $\bar{c}$ on the graviton self-energy. As displayed in Fig. 4, four diagrams (a), (b), (c) and (d) contribute. In the Feynman-'t 


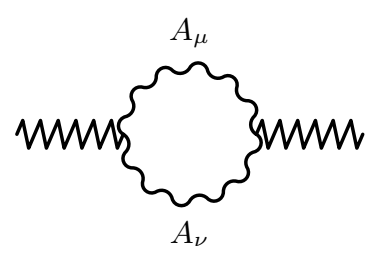

(a)

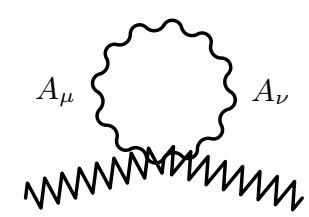

(b)

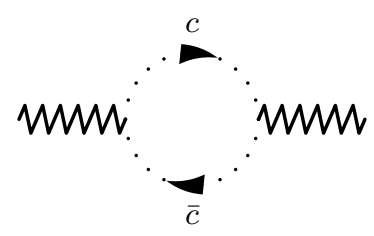

(c)

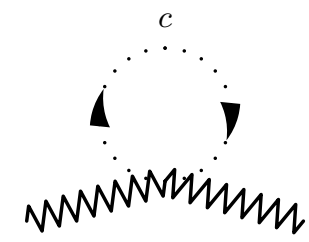

(d)

FIG. 4: Gauge- and ghost-field contributions to the graviton self-energy.

Hooft gauge $\xi_{G}=1$, these four diagrams may respectively be evaluated by the following integrals:

$$
\begin{aligned}
i \Pi_{4(a)}^{\mu \nu, \rho \sigma}(p) & =\frac{1}{2} \int \frac{d^{d} k}{(2 \pi)^{d}} V_{A A h}^{\mu \nu, \lambda, \delta}\left(k,-(p+k), m_{A}\right) V_{A A h}^{\rho \sigma, \alpha, \beta}\left(-k, p+k, m_{A}\right)\left[\frac{i \eta_{\alpha \lambda}}{k^{2}-m_{A}^{2}}\right]\left[\frac{i \eta_{\beta \gamma}}{(p+k)^{2}-m_{A}^{2}}\right], \\
i \Pi_{4(b)}^{\mu \nu, \rho \sigma}(p) & =\frac{1}{2} \int \frac{d^{d} k}{(2 \pi)^{d}} V_{A A h h}^{\mu \nu, \rho \sigma, \lambda, \delta}\left(k,-k, m_{A}^{2}\right)\left[\frac{i \eta_{\lambda \delta}}{k^{2}-m_{A}^{2}}\right], \\
i \Pi_{4(c)}^{\mu \nu, \rho \sigma}(p) & =-\int \frac{d^{d} k}{(2 \pi)^{d}} V_{\bar{c} c h}^{\mu \nu}\left(k,-(p+k), m_{A}\right) V_{\bar{c} c h}^{\rho \sigma}\left(-k, p+k, m_{A}\right)\left[\frac{i}{k^{2}-m_{A}^{2}}\right]\left[\frac{i}{(p+k)^{2}-m_{A}^{2}}\right], \\
i \Pi_{4(d)}^{\mu \nu, \rho \sigma}(p) & =-\int \frac{d^{d} k}{(2 \pi)^{d}} V_{H H h h}^{\mu \nu \rho \sigma}\left(k,-k, m_{A}\right)\left[\frac{i}{k^{2}-m_{A}^{2}}\right] .
\end{aligned}
$$

As done before, we proceed by including only the $\mathrm{CT} \delta \Lambda$ of the cosmological constant. Then, the form factors are given by

$$
\begin{aligned}
& F_{1}\left(p^{2}\right)=\frac{\kappa^{2}}{1800(4 \pi)^{2}\left(p^{2}\right)^{2}}\left[\left(\alpha_{1}+\alpha_{4}\right) B_{0}\left(p^{2}, m_{A}^{2}, m_{A}^{2}\right)+\left(\alpha_{2}+\alpha_{5}\right) A_{0}\left(m_{H}^{2}\right)+\left(\alpha_{3}+\alpha_{6}\right)\right] \\
& F_{2}\left(p^{2}\right)=\frac{\kappa^{2}}{1800(4 \pi)^{2}}\left(\alpha_{1} B_{0}\left(p^{2}, m_{A}^{2}, m_{A}^{2}\right)+\alpha_{2} A_{0}\left(m_{A}^{2}\right)+\alpha_{3}\right) \\
& F_{3}\left(p^{2}\right)=\frac{\kappa^{2}}{3600(4 \pi)^{2}}\left(\alpha_{4} B_{0}\left(p^{2}, m_{A}^{2}, m_{A}^{2}\right)+\alpha_{5} A_{0}\left(m_{A}^{2}\right)+\alpha_{6}\right) \\
& F_{4}\left(p^{2}\right)=-\frac{\kappa^{2}}{1800(4 \pi)^{2} p^{2}}\left(\alpha_{1} B_{0}\left(p^{2}, m_{A}^{2}, m_{A}^{2}\right)+\alpha_{2} A_{0}\left(m_{A}^{2}\right)+\alpha_{3}\right) \\
& F_{5}\left(p^{2}\right)=-\frac{\kappa^{2}}{3600(4 \pi)^{2} p^{2}}\left(\alpha_{4} B_{0}\left(p^{2}, m_{A}^{2}, m_{A}^{2}\right)+\alpha_{5} A_{0}\left(m_{A}^{2}\right)+\alpha_{6}\right)
\end{aligned}
$$

with

$$
\begin{aligned}
& \alpha_{1}=30\left(4 m_{A}^{4}-12 m_{A}^{2} p^{2}-\left(p^{2}\right)^{2}\right), \\
& \alpha_{2}=60\left(p^{2}-2 m_{A}^{2}\right), \\
& \alpha_{3}=120 m^{4}-530 m^{2} p^{2}+13\left(p^{2}\right)^{2}, \\
& \alpha_{4}=30\left[8 m_{A}^{4}+16 m_{A}^{2} p^{2}+3\left(p^{2}\right)^{2}\right], \\
& \alpha_{5}=-60\left(4 m_{A}^{2}+3 p^{2}\right), \\
& \alpha_{6}=240 m_{A}^{4}+590 m_{A}^{2} p^{2}-99\left(p^{2}\right)^{2} .
\end{aligned}
$$

The form factors become UV finite, after considering the CT effective action

$$
\Delta S_{A}=-\int d^{4} x \frac{\sqrt{-g}}{(4 \pi)^{2} \bar{\epsilon}}\left[\frac{m_{A}^{2}}{3} R-\frac{1}{30} R^{2}+\frac{1}{10} R^{\mu \nu} R_{\mu \nu}\right]
$$

Our result in (IV.27) agrees with [14] in the limit $m_{A} \rightarrow 0$. As with the scalar and fermion cases, gauge and ghost field contributions to the form factors $F_{1,2, \ldots, 5}$ satisfy the transversality relations stated in (IV.7). 


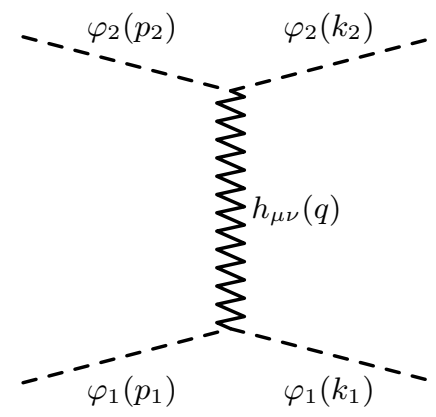

FIG. 5: The tree level scattering diagram.

\section{IV.5. Summary of Results}

Even though our calculations pertain to the gauged Abelian Higgs model, the results we presented here for the graviton self-energy have a general applicability. At the one-loop order, only the kinetic part of the matter Lagrangian contributes, whereas the part associated with matter interactions only enters at two loops. Thus, at the one-loop level, we only need to know the matter field content of the theory in terms of scalar, fermionic and gauge degrees of freedom. As a consequence, the total renormalized graviton self-energy tensor in a given theory may be summarized as follows:

$$
\Pi_{\mathrm{R}}^{\mu \nu, \rho \sigma}(p)=\sum_{i=1}^{N_{0}} \Pi_{0}^{\mu \nu, \rho \sigma}\left(p, m_{0, i}\right)+\sum_{i=1}^{N_{\frac{1}{2}}} \Pi_{\frac{1}{2}}^{\mu \nu, \rho \sigma}\left(p, m_{\frac{1}{2}, i}\right)+\sum_{i=1}^{N_{1}} \Pi_{1}^{\mu \nu, \rho \sigma}\left(p, m_{1, i}\right),
$$

where $\Pi_{0}^{\mu \nu, \rho \sigma}(p, m), \Pi_{\frac{1}{2}}^{\mu \nu, \rho \sigma}(p, m)$, and $\Pi_{1}^{\mu \nu, \rho \sigma}(p, m)$ denote the scalar, fermion, and gauge- and ghost-field contributions to the graviton self-energy with a generic mass $m$, respectively. Correspondingly, $N_{0}, N_{\frac{1}{2}}$ and $N_{1}$ denote the number of scalars, fermions, and gauge bosons. As the total self-energy is the sum of individual contributions which satisfy the transversality condition (IV.5), it is evident that the graviton remains massless at the one-loop level. Beyond the one loop, the validity of the GLET (III.50) and the diffeomorphisms WI (II.34) play a central role to preserve the property of masslessness of the graviton field to all orders in perturbation theory.

\section{MATTER QUANTUM CORRECTIONS TO THE NEWTONIAN POTENTIAL}

Having computed the matter effects on the graviton self-energy, we can now proceed to study the one-loop quantum corrections to the Newtonian potential $V(r)$. As we will see in this section, the radiatively corrected Newtonian potential can be derived from the $S$-matrix element describing the elastic scattering of two massive particles $\varphi_{1}$ and $\varphi_{2}$ in the non-relativistic limit. We will use these results to determine the long and short range limits of $V(r)$ and comment on their relevance. In the massless limit of loop particles, the known results for $V(r)$ stated in the introduction are reproduced.

For definiteness, we consider the scattering process $\varphi_{1}\left(p_{1}\right) \varphi_{2}\left(p_{2}\right) \rightarrow \varphi_{1}\left(k_{1}\right) \varphi_{2}\left(k_{2}\right)$, where $\varphi_{1}$ and $\varphi_{2}$ are two different gauge-singlet scalars. The action describing the interaction of $\varphi_{1,2}$ with gravity is given by

$$
S_{\varphi}=\frac{1}{2} \int d^{4} x \sqrt{-g}\left(g^{\mu \nu} \partial_{\mu} \varphi_{1} \partial_{\nu} \varphi_{1}-m_{1}^{2} \varphi_{1}^{2}+g^{\mu \nu} \partial_{\mu} \varphi_{2} \partial_{\nu} \varphi_{2}-m_{2}^{2} \varphi_{2}^{2}\right)
$$

where $m_{1}$ and $m_{2}$ are the masses of the fields $\varphi_{1}$ and $\varphi_{2}$, respectively. We shall use this action to derive the Feynman rules for the interactions of $\varphi_{1}$ and $\varphi_{2}$ to the graviton field $h_{\mu \nu}$. The analytical expressions for the relevant vertices are given in Appendix A

\section{V.1. Tree-Level Newtonian Potential}

It is instructive to briefly review how the classical Newtonian potential can be inferred from the tree-level $S$-matrix element of a given process $\varphi_{1}\left(p_{1}\right) \varphi_{2}\left(p_{2}\right) \rightarrow \varphi_{1}\left(k_{1}\right) \varphi_{2}\left(k_{2}\right)$. As illustrated in Fig. 5 , such a process proceeds at the tree level via the exchange of a single graviton in the $t$-channel. The momentum space amplitude $\mathcal{M}_{\text {tree }}$ is given by

$$
i \mathcal{M}_{\text {tree }}=i V_{\varphi_{1} \varphi_{1} h}^{\mu \nu}\left(p_{1},-k_{1}\right) \Delta_{0, \mu \nu, \rho \sigma}(q) V_{\varphi_{2} \varphi_{2} h}^{\rho \sigma}\left(p_{2},-k_{2}\right)
$$


where $q_{\mu}=\left(p_{1}-k_{1}\right)_{\mu}=\left(p_{2}-k_{2}\right)_{\mu}$ is the four-momentum of the graviton, $V_{\varphi_{1} \varphi_{1} h}^{\mu \nu}$ and $V_{\varphi_{2} \varphi_{2} h}^{\rho \sigma}$ are the tree-level vertex functions for the $\varphi_{1} \varphi_{1} h$ and $\varphi_{2} \varphi_{2} h$ vertices, respectively, and $\Delta_{0, \mu \nu, \rho \sigma}(q)$ is the tree-level graviton propagator.

Let us briefly discuss the gauge independence of the tree-level graph. The graviton propagator depends on the gauge fixing parameters $\xi_{D}$ and $\sigma$. However, as a consequence of the Ward identity of diffeomorphisms, the vertex functions $V_{\varphi_{1} \varphi_{1} h}^{\mu \nu}\left(p_{1},-k_{1}\right)$ and $V_{\varphi_{2} \varphi_{2} h}^{\mu \nu}\left(p_{2},-k_{2}\right)$ satisfy:

$$
\left(p_{1}-k_{1}\right)_{\mu} V_{\varphi_{1} \varphi_{1} h}^{\mu \nu}\left(p_{1},-k_{1}\right)=0, \quad\left(p_{2}-k_{2}\right)_{\mu} V_{\varphi_{2} \varphi_{2} h}^{\mu \nu}\left(p_{2},-k_{2}\right)=0,
$$

when the scalar fields are taken to be on-shell. As all dependence on the gauge-fixing parameters $\xi_{D}$ and $\sigma$ is carried by terms proportional to the longitudinal four-momentum $q_{\mu}$ (see Appendix A for an expression for the graviton propagator), all $\xi_{D^{-}}$and $\sigma$-dependent terms vanish thanks to $(\mathrm{V} .3)$, thus yielding a gauge-invariant result under the group of diffeomorphisms. In fact, this is equivalent to replacing the graviton propagator with the propagator in the gauge $\xi_{D}=\frac{1}{2}, \sigma=1$ (known as the harmonic or de Donder gauge):

$$
\Delta_{0, \mu \nu, \rho \sigma}(q) \rightarrow \frac{P_{\mu \nu \rho \sigma}}{q^{2}+i \epsilon} .
$$

The non-relativistic limit of the amplitude $\mathcal{M}_{\text {tree }}$ is obtained by expanding in the three-momenta of the external fields and considering only terms that diverge in the IR limit of vanishing 3-momenta. These terms have been called non-analytic terms in [30]. Expanding the tree-level vertex functions, $V_{\varphi_{1} \varphi_{1} h}$ and $V_{\varphi_{2} \varphi_{2} h}$, about the three-momenta of the external particles, we obtain the leading terms of the expansion:

$$
V_{\varphi_{1} \varphi_{1} h}^{\mu \nu}=i \kappa m_{1}^{2} \delta_{0}^{\mu} \delta_{0}^{\nu} ; \quad V_{\varphi_{2} \varphi_{2} h}^{\mu \nu}=i \kappa m_{2}^{2} \delta_{0}^{\mu} \delta_{0}^{\nu} .
$$

Employing the elementary identities given in $(\mathrm{V} .3)$, the tree-level amplitude $\mathcal{M}_{\text {tree }}$ in $(\mathrm{V} .2)$ takes on the simple form in the non-relativistic limit:

$$
\mathcal{M}_{\text {tree }}=-\frac{\kappa^{2} m_{1}^{2} m_{2}^{2}}{|\vec{q}|^{2}}
$$

where $|\vec{q}|=\left|\vec{p}_{1}-\vec{k}_{1}\right|$ is the 3-momentum of the exchange graviton.

To derive the Newtonian potential from the scattering amplitude $\mathcal{M}_{\text {tree }}$, we use the relation [30]:

$$
V(\vec{r})=\frac{1}{2 m_{1}} \frac{1}{2 m_{2}} \int \frac{d^{3} q}{(2 \pi)^{3}} e^{i \vec{q} \cdot \vec{r}} \mathcal{M}_{\text {tree }}(\vec{q}) .
$$

Note that the factors $1 / 2 m_{1}$ and $1 / 2 m_{2}$ result from the normalization of single particle states. Using the definition $\kappa^{2}=16 \pi G$ and the well-known result for the integral

$$
\int \frac{d^{3} q}{(2 \pi)^{3}} e^{i \vec{q} \cdot \vec{r}} \frac{1}{|\vec{q}|^{2}}=\frac{1}{4 \pi r}
$$

we obtain the scattering potential

$$
V(r)=-\frac{G m_{1} m_{2}}{r},
$$

which is the classical Newtonian potential. Notice that $V(r)$ has been obtained by pure quantum field-theoretic means, and is manifestly gauge invariant and process independent, i.e. the same result would have been obtained, if we had considered fermions or vector bosons, instead of scalars, as external particles.

\section{V.2. Matter Quantum Corrections}

We shall now compute the one-loop matter quantum corrections to the scattering process $\varphi_{1} \varphi_{2} \rightarrow \varphi_{1} \varphi_{2}$, shown in Fig. 5. Given that $\varphi_{1}$ and $\varphi_{2}$ are gauge singlets, only self-energy effects contribute to this process, as illustrated in Fig. 6. If these scalar fields were charged under a $U(1)$ gauge group, one must also include vertex and box contributions. The case of an elastic scattering with charged scalars was studied in [29, whilst the scattering process with external charged fermions under $U(1)$ was analyzed in [32. However, we note that quantum effects on the Newtonian potential do not depend on the specific nature of the external scattered particles, i.e. the quantum effects are process independent.

We should remark here that the use of an one-loop resummed graviton propagator proves necessary. A conventional perturbative expansion in terms of graviton self-energies produces corrections to the potential which are linear in the separation i.e. $\propto r$, when the loop mass is non-zero. This contribution diverges as $r \rightarrow \infty$. We shall show that only a calculation of the potential based on the resummed graviton propagator gives the correct asymptotic behaviour. 


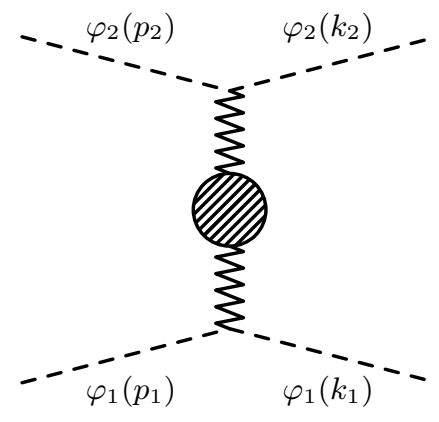

FIG. 6: The class of diagrams corresponding to matter effects.

\section{V.2.1. The amplitude and its non-relativistic limit}

The one-loop transition amplitude shown in Fig. 6 is given by

$$
i \mathcal{M}_{1-\text { loop }}=i V_{\varphi_{1} \varphi_{1} h}^{\mu \nu}\left(p_{1},-k_{1}\right) \Delta_{\mu \nu, \rho \sigma}(q) V_{\varphi_{2} \varphi_{2} h}^{\rho \sigma}\left(p_{2},-k_{2}\right) .
$$

where $\Delta_{\mu \nu, \rho \sigma}(q)$ is the resummed graviton propagator. To achieve this resummation at one-loop order, we must resum the Dyson series of the one-loop graviton self-energy graphs. Specifically, the resummed graviton propagator $\Delta_{\mu \nu, \rho \sigma}(q)$ is defined by the equation

$$
\left(\Delta_{0}^{-1 \mu \nu, \alpha \beta}(q)+\Pi_{\mathrm{R}}^{\mu \nu, \alpha \beta}(q)\right) \Delta_{\alpha \beta, \rho \sigma}(q)=\frac{1}{2}\left(\delta_{\mu}^{\rho} \delta_{\nu}^{\sigma}+\delta_{\mu}^{\sigma} \delta_{\nu}^{\rho}\right)
$$

Here, $\Delta_{0}^{-1 \mu \nu, \rho \sigma}(q)$ is the tree-level inverse propagator and $\Pi_{\mathrm{R}}^{\mu \nu \rho \sigma}(q)$ is the renormalized graviton self-energy which has been calculated explicitly in Section IV.

In order to invert the relation (V.11), we first write the resummed graviton propagator in terms of its possible form factors:

$$
\begin{aligned}
\Delta_{\mu \nu, \rho \sigma}(q)= & q_{\mu} q_{\nu} q_{\rho} q_{\sigma} \Delta_{1}\left(q^{2}\right)+\eta_{\mu \nu} \eta_{\rho \sigma} \Delta_{2}\left(q^{2}\right)+\left(\eta_{\mu \rho} \eta_{\nu \sigma}+\eta_{\nu \rho} \eta_{\mu \sigma}\right) \Delta_{3}\left(q^{2}\right) \\
& +\left(\eta_{\mu \nu} q_{\rho} q_{\sigma}+\eta_{\rho \sigma} q_{\mu} q_{\nu}\right) \Delta_{4}\left(p^{2}\right)+\left(\eta_{\mu \rho} q_{\nu} q_{\sigma}+\eta_{\nu \rho} q_{\mu} q_{\sigma}+\eta_{\mu \sigma} q_{\nu} q_{\rho}+\eta_{\nu \sigma} q_{\mu} q_{\rho}\right) \Delta_{5}\left(q^{2}\right)
\end{aligned}
$$

Employing the method of orthogonal projectors, we find

$$
\begin{aligned}
& \Delta_{1}\left(q^{2}\right)=\frac{4}{3\left(q^{2}\right)^{2}\left(q^{2}-4 F_{3}\left(q^{2}\right)\right)}-\frac{4}{3\left(q^{2}\right)^{2}\left(q^{2}+3 F_{2}\left(q^{2}\right)+2 F_{3}\left(q^{2}\right)\right)}, \\
& \Delta_{2}\left(q^{2}\right)=-\frac{2}{3\left(q^{2}-4 F_{3}\left(q^{2}\right)\right)}-\frac{1}{3\left(q^{2}+3 F_{2}\left(q^{2}\right)+2 F_{3}\left(q^{2}\right)\right)}, \\
& \Delta_{3}\left(q^{2}\right)=\frac{1}{\left(q^{2}-4 F_{3}\left(q^{2}\right)\right)}, \\
& \Delta_{4}\left(q^{2}\right)=\frac{2}{3 q^{2}\left(q^{2}-4 F_{3}\left(q^{2}\right)\right)}-\frac{1}{3 q^{2}\left(q^{2}+3 F_{2}\left(q^{2}\right)+2 F_{3}\left(q^{2}\right)\right)} \\
& \Delta_{5}\left(q^{2}\right)=\frac{1}{\left(q^{2}\right)^{2}}-\frac{1}{q^{2}\left(q^{2}-4 F_{3}\left(q^{2}\right)\right)}
\end{aligned}
$$

where $F_{2}, F_{3}$ are the graviton self-energy form factors defined in (IV.6).

Let us now discuss the gauge dependence of this amplitude. Writing out the full Dyson series for the resummed propagator, we obtain

$$
\Delta_{\mu \nu, \rho \sigma}=\Delta_{0 \mu \nu, \rho \sigma}-\Delta_{0 \mu \nu, \alpha \beta} \Pi_{\mathrm{R}}^{\alpha \beta, \gamma \delta} \Delta_{0 \gamma \delta, \rho \sigma}+\Delta_{0 \mu \nu, \alpha \beta} \Pi_{\mathrm{R}}^{\alpha \beta, \gamma \delta} \Delta_{0 \gamma \delta, \lambda \kappa} \Pi_{\mathrm{R}}^{\lambda \kappa, \epsilon \zeta} \Delta_{0 \epsilon \zeta, \rho \sigma}+\cdots
$$

Given that the tree level propagators must contract with either the tree level vertex functions $V_{\varphi_{1} \varphi_{1} h}^{\mu \nu}$ or $V_{\varphi_{2} \varphi_{2} h}^{\mu \nu}$, where the scalars are on-shell, or the renormalized graviton self-energy $\Pi_{\mathrm{R}}^{\mu \nu, \rho \sigma}$, any term in the propagator which explicitly depends on components of the longitudinal four-momenta $q_{\mu}$ will vanish due to the identities (V.3) and (IV.5). 
As a consequence, the one-loop transition amplitude $\mathcal{M}_{1-\text { loop }}$ becomes independent of the gauge-fixing parameters $\xi_{D}$ and $\sigma$ of the diffeomorphisms. Like the tree-level case, we can use the harmonic gauge for the graviton propagator [cf. (V.4] ] to simplify the calculation.

In the non-relativistic limit, the one-loop amplitude becomes

$$
\mathcal{M}_{1-\text { loop }}=-\kappa^{2} m_{1}^{2} m_{2}^{2}\left[\frac{4}{3}\left(\frac{1}{|\vec{q}|^{2}+4 F_{3}\left(-|\vec{q}|^{2}\right)}\right)+\frac{1}{3}\left(\frac{1}{3 F_{2}\left(-|\vec{q}|^{2}\right)+2 F_{3}\left(-|\vec{q}|^{2}\right)-|\vec{q}|^{2}}\right)\right] .
$$

This amplitude diverges as $|\vec{q}| \rightarrow 0$, since both the form factors $F_{2}$ and $F_{3}$ vanish in this limit, thanks to (IV.7). This singularity of the transition amplitude $\mathcal{M}_{1-\text { loop as }}|\vec{q}| \rightarrow 0$ is a simple manifestation of the masslessness of the graviton field. When going to the non-relativistic limit, the presence of a particle with mass $m$ in the loop requires special care, as $m$ is another dimensionful parameter entering the calculation of the amplitude. In this case, one needs to proceed carefully and compare the size of $|\vec{q}|$ to $m$, rather than simply taking the IR limit $|\vec{q}| \ll 1$. In fact, one has to distinguish between three possible cases for a loop particle with mass $m$ : $|\vec{q}| \gg m,|\vec{q}| \sim m$ and $|\vec{q}| \ll m$. In the calculation that follows, we first compute the potential in the general case, before translating the aforementioned three limits into position space.

\section{V.2.2. Computation of the scattering potential}

Our aim is now to compute the Newtonian potential from the one-loop transition amplitude. As before, we may define the Newtonian potential in close analogy to (V.7), which may be represented by the one-dimensional integral of the Fourier transform:

$$
V(r)=-\frac{i}{(2 \pi)^{2}} \int_{-\infty}^{\infty} d q\left(\frac{q}{r} e^{i q r} \widehat{\mathcal{M}}_{1-\text { loop }}(q)\right)
$$

where $q \equiv|\vec{q}|$ and $\widehat{\mathcal{M}}_{1-\text { loop }} \equiv \frac{1}{2 m_{1}} \frac{1}{2 m_{2}} \mathcal{M}_{1-\text { loop}}$. The above expression $(\mathrm{V} .20$ includes the tree-level contribution to the potential, as well as the one-loop matter quantum corrections, through the resummed graviton propagator.

In order to perform the integration, we analytically continue $q$ to a complex variable and integrate over a closed contour in the complex plane which includes the integral of interest V.20). Given that the value of the closed contour integral depends upon the residue of the poles within the contour, we begin by identifying the poles of the integrand. Explicitly, we find that there are three real poles for the resummed graviton propagator: the standard one at $q=0$ and two others that occur in the Planck mass range at $q= \pm q_{0}$, where $q_{0} \sim M_{\mathrm{P}}$. The latter poles signify the breakdown of perturbative quantum gravity and therefore we call them Landau poles.

An analytic expression for the square $q_{0}^{2}$ of the Landau poles may be determined by searching for non-zero roots of the denominators in $\left(\mathrm{V} .19\right.$. Assuming the loop masses are small compared to $1 / \kappa^{2}=M_{\mathrm{P}}^{2}$, we may expand the root in powers of $\kappa^{2}$ and its inverse. It can then be shown that the Landau pole diverges as $\kappa^{2} \rightarrow 0$ and that the pole is a simple pole. Thus, the leading term in the expansion is the term proportional to $1 / \kappa^{2}$. Hence, we obtain the approximate analytic expression for $q_{0}^{2}$ :

$$
q_{0}^{2}=\frac{1920 \pi^{2}}{\kappa^{2} \beta}\left[W\left(\frac{1920 \pi^{2} \exp (-\gamma / \beta)}{\kappa^{2} \mu^{2} \beta}\right)\right]^{-1}
$$

with $\beta=N_{0}+3 N_{\frac{1}{2}}+14 N_{1}$ and $\gamma=\frac{2}{15}\left(23 N_{0}+59 N_{\frac{1}{2}}+142 N_{1}\right)$, where $N_{s}$ is the number of fields of spin $s=$ 0 (scalar), $\frac{1}{2}$ (Weyl fermion), 1 (vector boson) and $W(z)$ is the Lambert $W$-function defined by the inverse relation: $z=W(z) e^{W(z)}$.

One may wonder whether there are other complex poles, in addition to the three real poles mentioned above. To address this question, we use the argument principle, which states that, for some complex function $f(z)$, it holds

$$
\frac{1}{2 \pi i} \oint_{\gamma} \frac{f^{\prime}(z)}{f(z)} d z=N-P
$$

where $N$ is the number of roots of $f(z), P$ is the number of poles of $f(z)$, and $\gamma$ is a closed contour which contains the entire complex plane whilst excluding the branch cuts of the function. The integrand $q e^{-q r} \widehat{\mathcal{M}}_{1-\text { loop }}(q)$ may be split into two parts $f_{1}(q)$ and $f_{2}(q)$ :

$$
f_{1}(q)=-\frac{\kappa^{2} m_{1} m_{2}}{3}\left(\frac{q e^{-q r}}{-q^{2}+4 F_{3}\left(q^{2}\right)}\right), \quad f_{2}(q)=-\frac{\kappa^{2} m_{1} m_{2}}{12}\left(\frac{q e^{-q r}}{3 F_{2}\left(q^{2}\right)+2 F_{3}\left(q^{2}\right)+q^{2}}\right)
$$




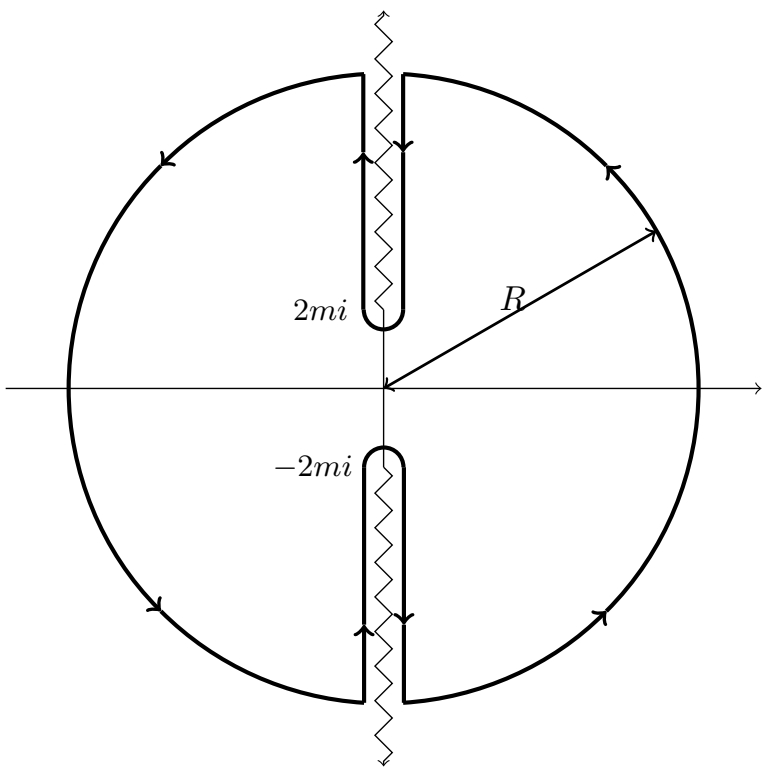

FIG. 7: The contour used for the complex integral in $(\mathrm{V} .22$ to compute the number of poles and roots for a single loop mass $m$. This contour covers the whole complex plane as $R \rightarrow \infty$, whilst excluding the two branch cuts indicated by the zigzag lines.

as there are two terms in (V.19). We now observe that, for every matter field in the loop with mass $m$, there are two branch cuts in the complex plane for $f_{1}(q)$ and $f_{2}(q)$. The first branch cut is along the positive imaginary interval $[2 m i,+i \infty)$, whilst the second one is along the negative imaginary interval $[-2 m i,-i \infty)$. Taking these two branch cuts into account, we may determine $N-P$ for both functions independently, using the contour $\gamma$ depicted in Fig. 7 . In both cases, we obtain

$$
N-P=-3 .
$$

Since the form factors $F_{2}\left(q^{2}\right), F_{3}\left(q^{2}\right)$ do not diverge for finite values of $q, f_{1}(q)$ and $f_{2}(q)$ have no roots. This gives $P=3$ for both functions. Substituting the expression for the Landau poles (V.21) into the denominator of each function, we obtain zero in both cases when the loop masses are small compared to $M_{P}$. Therefore, both functions diverge at the same points: the real pole at $q=0$ and the two Landau poles $q^{2}=q_{0}^{2}$. Consequently, the resummed graviton propagator and so $\widehat{\mathcal{M}}_{1-\text { loop }}(q)$ has no other complex poles that we need to worry about.

Knowing the location the three real poles, we may construct a closed contour to compute the Fourier transform (V.20), which is illustrated in Fig. 8. By means of this contour, we may evaluate the potential as follows:

$$
V(r)=V_{\text {res }}(r)+V_{\text {branch }}(r),
$$

where

$$
\begin{aligned}
V_{\text {res }}(r) & =\frac{1}{2 \pi r} \sum_{n} \operatorname{Res}\left(q e^{i q r} \widehat{\mathcal{M}}_{1-\text { loop }}(q), q_{n}\right)-\frac{i}{(2 \pi)^{2} r} \sum_{i=1}^{3} \lim _{\epsilon_{i} \rightarrow 0^{+}} \int_{\gamma_{\epsilon_{i}}} d q\left(q e^{i q r} \widehat{\mathcal{M}}_{1-\text { loop }}(q)\right), \\
V_{\text {branch }}(r) & =-\frac{1}{2 \pi^{2} r} \lim _{\epsilon \rightarrow 0^{+}} \int_{2 m}^{\infty} d q q e^{-q r} \operatorname{Im}\left(\widehat{\mathcal{M}}_{1-\text { loop }}(i q+\epsilon)\right),
\end{aligned}
$$

and $\operatorname{Res}\left(q e^{i q r} \widehat{\mathcal{M}}_{1-\text { loop }}(q), q_{n}\right)$ stands for the residue of a given complex pole $q_{n}$. The summation in the first term of $V_{\text {res }}(r)$ is taken over all complex poles, $q_{n}$, of $q e^{i q r} \widehat{\mathcal{M}}_{1-\text { loop }}(q)$. There are no contributions from the $\gamma_{R 1}$ and $\gamma_{R 2}$ contours, as they vanish as the radius of the contour $R$ goes to infinity. We note that for a radius $R$ bigger than the size of the Landau pole $q_{0}$, the contributions $\gamma_{\epsilon_{1}}$ and $\gamma_{\epsilon_{3}}$ must be included.

Let us first analyze the residue at the physical pole $q=0$ for the resummed one-loop amplitude. This is given by

$$
\operatorname{Res}\left(q e^{i q r} \widehat{\mathcal{M}}_{1-\text { loop }}(q), 0\right)=-\alpha \frac{\kappa^{2} m_{1} m_{2}}{4},
$$




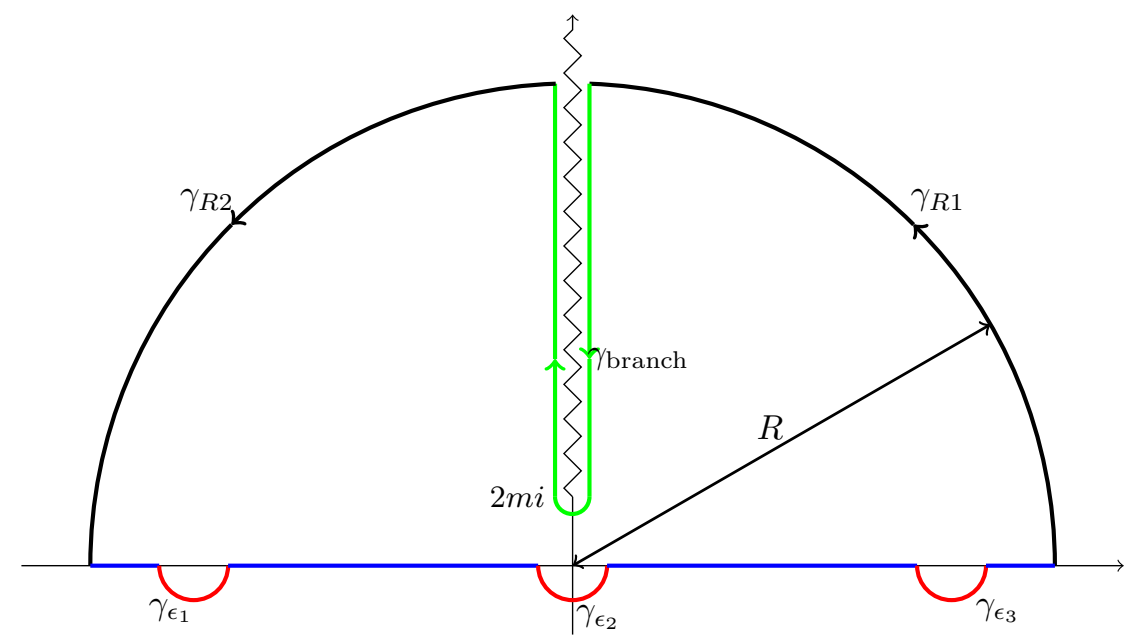

FIG. 8: The contour used to compute the Fourier transform in $(\mathrm{V} .20$. For a generic non-zero loop mass $m$, there is a branch cut that starts at $2 m i$ and extends to $i \infty$ as illustrated by the zigzag line.

where

$$
\alpha=\frac{1}{4}\left[\frac{4}{3}\left(1-4 \sum_{i=1}^{n} a_{i}\right)^{-1}-\frac{1}{3}\left(1+2 \sum_{i=1}^{n} a_{i}+3 \sum_{i=1}^{n} b_{i}\right)^{-1}\right]
$$

with

$$
a_{i}=\left.\frac{\partial F_{2, i}\left(q^{2}\right)}{\partial q^{2}}\right|_{q^{2}=0}, \quad b_{i}=\left.\frac{\partial F_{3, i}\left(q^{2}\right)}{\partial q^{2}}\right|_{q^{2}=0} .
$$

In the above, $F_{j, i}$ is the $j$ th form factor of the $i$ th field and $n$ is the number of fields. The formulae for $a_{i}$ and $b_{i}$ derived from $\left(\mathrm{V} .30\right.$ only hold if the form factors have non-zero loop mass (or are analytic in $q^{2}$ ). If all particle masses in the loops vanish, we have $a_{i}=b_{i}=0$, implying that $\alpha=1$.

As for the residues of the Landau poles, we shall not include them in the calculation, as these are related with the potential UV completion of the theory of quantum gravity. The simplest way to achieve this is to introduce a UV cut-off just below the Landau pole $q_{0}$ in the Fourier transform $(\mathrm{V} .20)$. In this case, the contributions from the $\gamma_{R 1}$ and $\gamma_{R 2}$ contours will not vanish, but the cut-off integral will differ by terms of $O\left(\mathrm{~m} / q_{0}\right)$ in comparison to the other $O(1)$ terms. Therefore, we may safely ignore these cut-off suppressed contributions in favour of the other leading terms of order one.

We may now compute $V_{\text {res }}(r)$, using the result for the residue in $(\mathrm{V} .28)$. Computing the remaining contour integral $\gamma_{\epsilon_{2}}$ in V.26 gives rise to the potential

$$
V_{\mathrm{res}}(r)=-\alpha \frac{G m_{1} m_{2}}{r}
$$

Evidently, this is a rescaled version of the Newtonian potential. Specifically, for a scalar field of mass $m_{H}$, we have

$$
a_{H}=\left.\frac{\partial F_{2, H}\left(p^{2}\right)}{\partial p^{2}}\right|_{p^{2}=0}=\frac{\kappa^{2} m_{H}^{2}}{384 \pi^{2}}\left[\ln \left(\frac{m_{H}^{2}}{\mu^{2}}\right)-1\right], \quad b_{H}=\left.\frac{\partial F_{3, H}\left(p^{2}\right)}{\partial p^{2}}\right|_{p^{2}=0}=-\frac{\kappa^{2} m_{H}^{2}}{768 \pi^{2}}\left[\ln \left(\frac{m_{H}^{2}}{\mu^{2}}\right)-1\right] .
$$

For a fermion of mass $m_{\psi}$, we obtain

$$
a_{\psi}=\left.\frac{\partial F_{2, \psi}\left(p^{2}\right)}{\partial p^{2}}\right|_{p^{2}=0}=\frac{\kappa^{2} m_{\psi}^{2}}{192 \pi^{2}}\left[\ln \left(\frac{m_{\psi}^{2}}{\mu^{2}}\right)-1\right], \quad b_{\psi}=\left.\frac{\partial F_{3, \psi}\left(p^{2}\right)}{\partial p^{2}}\right|_{p^{2}=0}=-\frac{\kappa^{2} m_{\psi}^{2}}{384 \pi^{2}}\left[\ln \left(\frac{m_{\psi}^{2}}{\mu^{2}}\right)-1\right] .
$$

Finally, for a massive gauge field of mass $m_{A}$ (without ghosts), we find

$$
a_{A}=\left.\frac{\partial F_{2, A}\left(p^{2}\right)}{\partial p^{2}}\right|_{p^{2}=0}=-\frac{\kappa^{2} m_{A}^{2}}{192 \pi^{2}}\left[\ln \left(\frac{m_{A}^{2}}{\mu^{2}}\right)-2\right], \quad b_{A}=\left.\frac{\partial F_{3, A}\left(p^{2}\right)}{\partial p^{2}}\right|_{p^{2}=0}=\frac{\kappa^{2} m_{A}^{2}}{384 \pi^{2}}\left[\ln \left(\frac{m_{A}^{2}}{\mu^{2}}\right)-2\right] .
$$

Astronomical observations can only measure the combination $\alpha G$, rather than $G$ alone, thus leading to a renormalization of the Newtonian constant $G$. However, we should note that the quantity $\alpha$ differs significantly from 1 when the loop masses are comparable to the Planck mass $M_{\mathrm{P}}$, which is a case that we will not be considering here. 


\section{V.2.3. The branch cut contribution}

Our next task is to compute the branch cut contribution $V_{\text {branch }}(r)$. To deal with the complexity of the integrand, we rewrite the one-loop corrected Newtonian potential as follows:

$$
V(r)=-\frac{G m_{1} m_{2}}{r}(\alpha+\Delta V(r))
$$

where the coefficient $\alpha$ given by $(\mathrm{V} .29$ pertains to the residue contributions and the dimensionless quantity $\Delta V(r)$ refers to the part of the potential resulting from the branch cut, i.e.

$$
V_{\text {branch }}(r)=-\frac{G m_{1} m_{2}}{r} \Delta V(r)
$$

We observe that the integral $V_{\text {branch }}(r)$ can be computed accurately by taking the first order term in a perturbative expansion in $\kappa^{2}$. To leading order in $\kappa^{2}$, the contributions to $V_{\text {branch }}(r)$ from scalar $(H)$, fermion $(\psi)$ and gauge boson $\left(A^{\mu}\right)$ loops may be calculated individually, such that $\Delta V(r)$ is given by the sum:

$$
\Delta V(r)=\Delta V_{H}(r)+\Delta V_{\psi}(r)+\Delta V_{A}(r)
$$

We will first present the calculation for the scalar loops and then simply state the results of the fermion and gauge fields. The branch cut effect due to a massive Higgs boson $H$ is given by the integral

$$
\Delta V_{H}(r)=\frac{G}{60 \pi} \int_{2 m}^{\infty} d q e^{-q r}\left(3-\frac{4 m_{H}^{2}}{q^{2}}+\frac{28 m_{H}^{4}}{q^{4}}\right) \sqrt{q^{2}-4 m_{H}^{2}},
$$

which is analytically calculable. Using the substitution $q=2 m_{H} \cosh x, \Delta V_{H}(r)$ may be rewritten as

$$
\Delta V_{H}(r)=\frac{G m_{H}^{2}}{15 \pi} \int_{0}^{\infty} d x e^{-2 m r \cosh x}\left(\cosh ^{2} x-1\right)\left(3-\operatorname{sech}^{2} x+\frac{7}{4} \operatorname{sech}^{4} x\right) .
$$

To proceed further, we first remind ourselves that the modified Bessel functions of the second kind $K_{\alpha}(\hat{r})$ have the integral representation

$$
K_{\alpha}(\hat{r})=\int_{0}^{\infty} d x e^{-\hat{r} \cosh x} \cosh (\alpha x)
$$

and so it is

$$
K_{0}(\hat{r})=\int_{0}^{\infty} d x e^{-\hat{r} \cosh x}
$$

Moreover, we can use the the hyperbolic trigonometric identity $\cosh ^{2} x=(1+\cosh 2 x) / 2$ to calculate the following integral:

$$
\int_{0}^{\infty} d x e^{-\hat{r} \cosh x} \cosh ^{2} x=\frac{1}{2}\left(K_{0}(\hat{r})+K_{2}(\hat{r})\right) .
$$

Apart from integrals containing $\cosh x$, there are also integrals involving sech $x$, defined as

$$
I_{n}(r)=\int_{0}^{\infty} d x e^{-r \cosh x} \operatorname{sech}^{n} x .
$$

We may compute the functions $I_{n}(r)$ recursively, by means of integration by parts. The relevant integrals of interest are

$$
\begin{aligned}
& I_{1}(r)=\int_{r}^{\infty} d x K_{0}(x)=-\frac{1}{2} \pi\left(r \boldsymbol{L}_{-1}(r) K_{0}(r)+r \boldsymbol{L}_{0}(r) K_{1}(r)-1\right) \\
& I_{2}(r)=r\left(K_{1}(r)-I_{1}(r)\right) \\
& I_{3}(r)=\frac{1}{2}\left(r K_{0}(r)-r I_{2}(r)+I_{1}(r)\right), \\
& I_{4}(r)=\frac{2}{3} I_{2}(r)+\frac{r}{3}\left(I_{1}(r)-I_{3}(r)\right),
\end{aligned}
$$


where $\boldsymbol{L}_{\alpha}(r)$ is the modified Struve function which has the integral representation

$$
\boldsymbol{L}_{\alpha}(r)=\frac{2^{1-\alpha} r^{\alpha}}{\sqrt{\pi} \Gamma\left(\alpha+\frac{1}{2}\right)} \int_{0}^{\frac{\pi}{2}} d x \sinh (r \cos x) \sin ^{2 \alpha} x
$$

for $\operatorname{Re}(\alpha)>-\frac{1}{2}$. The latter representation may be analytically continued to include other values of the index $\alpha$ of the modified Struve function $\boldsymbol{L}_{\alpha}(r)$.

We are now in a position to analytically compute the branch cut term $\Delta V_{H}(r)$ in terms of the modified Bessel and Struve functions. Defining the dimensionless parameter $\hat{r}_{H}=2 m_{H} r$, we obtain for the Higgs-scalar contribution:

$$
\begin{aligned}
\Delta V_{H}(r)=-\frac{G m_{H}^{2}}{360 \pi}[ & \frac{1}{2} \pi\left(7 \hat{r}_{H}^{2}-45\right) \hat{r}_{H}^{2}\left(\boldsymbol{L}_{-1}\left(\hat{r}_{H}\right) K_{0}\left(\hat{r}_{H}\right)+\boldsymbol{L}_{0}\left(\hat{r}_{H}\right) K_{1}\left(\hat{r}_{H}\right)\right)-\frac{7 \pi \hat{r}_{H}^{3}}{2}+\frac{45 \pi \hat{r}_{H}}{2} \\
& \left.+7 \hat{r}_{H}^{3} K_{1}\left(\hat{r}_{H}\right)-7 \hat{r}_{H}^{2} K_{0}\left(\hat{r}_{H}\right)-38 \hat{r}_{H} K_{1}\left(\hat{r}_{H}\right)+60 K_{0}\left(\hat{r}_{H}\right)-36 K_{2}\left(\hat{r}_{H}\right)\right]
\end{aligned}
$$

Similarly, for a Dirac fermion $\psi$, we define the dimensionless parameter $\hat{r}_{\psi}=2 m_{\psi} r$, in terms of which the branch-cut contribution reads:

$$
\begin{aligned}
\Delta V_{\psi}(r)=-\frac{G m_{\psi}^{2}}{180 \pi}[ & \frac{1}{2} \pi\left(7 \hat{r}_{\psi}^{2}-15\right) \hat{r}_{\psi}^{2}\left(\boldsymbol{L}_{-1}\left(\hat{r}_{\psi}\right) K_{0}\left(\hat{r}_{\psi}\right)+\boldsymbol{L}_{0}\left(\hat{r}_{\psi}\right) K_{1}\left(\hat{r}_{\psi}\right)\right)-\frac{7 \pi \hat{r}_{\psi}^{3}}{2}+\frac{15 \pi \hat{r}_{\psi}}{2} \\
& \left.+7 \hat{r}_{\psi}^{3} K_{1}\left(\hat{r}_{\psi}\right)-7 \hat{r}_{\psi}^{2} K_{0}\left(\hat{r}_{\psi}\right)-8 \hat{r}_{\psi} K_{1}\left(\hat{r}_{\psi}\right)-30 K_{0}\left(\hat{r}_{\psi}\right)+24 K_{2}\left(\hat{r}_{\psi}\right)\right]
\end{aligned}
$$

Finally, the branch-cut contribution arising from a $U(1)$ gauge boson $A_{\mu}$ and its associate ghost field is given by

$$
\begin{array}{r}
\Delta V_{A}(r)=-\frac{G m_{A}^{2}}{360 \pi}\left[\pi\left(7 \hat{r}_{A}^{2}+195\right) \hat{r}_{A}^{2}\left(\boldsymbol{L}_{-1}\left(\hat{r}_{A}\right) K_{0}\left(\hat{r}_{A}\right)+\boldsymbol{L}_{0}\left(\hat{r}_{A}\right) K_{1}\left(\hat{r}_{A}\right)\right)-7 \pi \hat{r}_{A}^{3}-195 \pi \hat{r}_{A}\right. \\
\left.+14 \hat{r}_{A}^{3} K_{1}\left(\hat{r}_{A}\right)-14 \hat{r}_{A}^{2} K_{0}\left(\hat{r}_{A}\right)+404 \hat{r}_{A} K_{1}\left(\hat{r}_{A}\right)-240 K_{0}\left(\hat{r}_{A}\right)-192 K_{2}\left(\hat{r}_{A}\right)\right]
\end{array}
$$

with $\hat{r}_{A}=2 m_{A} r$. We have checked that our perturbative analytical expressions for the branch cut contributions are in excellent agreement with numerical results derived by using the fully resummed graviton propagator to less than 1 part in $10^{-16}$. Plots of the different contributions to the potential for different loop masses are given in Fig. 9 . These plots demonstrate the exponential decay of loop effects due to particles with non-zero mass, as a function of distance $r$.

Using the above analytical results, it is not difficult to verify that the loop-corrected potential exhibits the desirable property:

$$
\lim _{r \rightarrow \infty} V(r)=0
$$

To see this explicitly, we use the large- $r$ asymptotic formulae for the modified Bessel and Struve functions:

$$
K_{\alpha}(r) \sim \sqrt{\frac{\pi}{2 r}} e^{-r}, \quad \boldsymbol{L}_{\alpha}(r) \sim \sqrt{\frac{1}{2 \pi r}} e^{r}-\frac{2^{1-\alpha} r^{\alpha-1}}{\sqrt{\pi} \Gamma\left(\alpha+\frac{1}{2}\right)} .
$$

In particular, for the scalar case, the branch-cut term $\Delta V_{H}(r)$ for $\hat{r}_{H} \gg 1$ simplifies to

$$
\Delta V_{H}(r)=-\frac{7 G m_{H}^{2}}{240 \sqrt{\pi m_{H}}}\left(\frac{e^{-2 m_{H} r}}{\sqrt{r}}-\frac{2}{3} m_{H} \sqrt{r} e^{-2 m_{H} r}\right) .
$$

For the fermion case, we have for $\hat{r}_{\psi} \gg 1$

$$
\Delta V_{\psi}(r)=\frac{7 G m_{\psi}^{2}}{60 \sqrt{\pi m_{\psi}}}\left(\frac{e^{-2 m_{\psi} r}}{\sqrt{r}}-\frac{2}{3} m_{\psi} \sqrt{r} e^{-2 m_{\psi} r}\right)
$$

and for the gauge boson case for $\hat{r}_{A} \gg 1$,

$$
\Delta V_{A}(r)=-\frac{7 G m_{A}^{2}}{120 \sqrt{\pi m_{A}}}\left(\frac{e^{-2 m_{A} r}}{\sqrt{r}}-\frac{2}{3} m_{A} \sqrt{r} e^{-2 m_{A} r}\right) .
$$



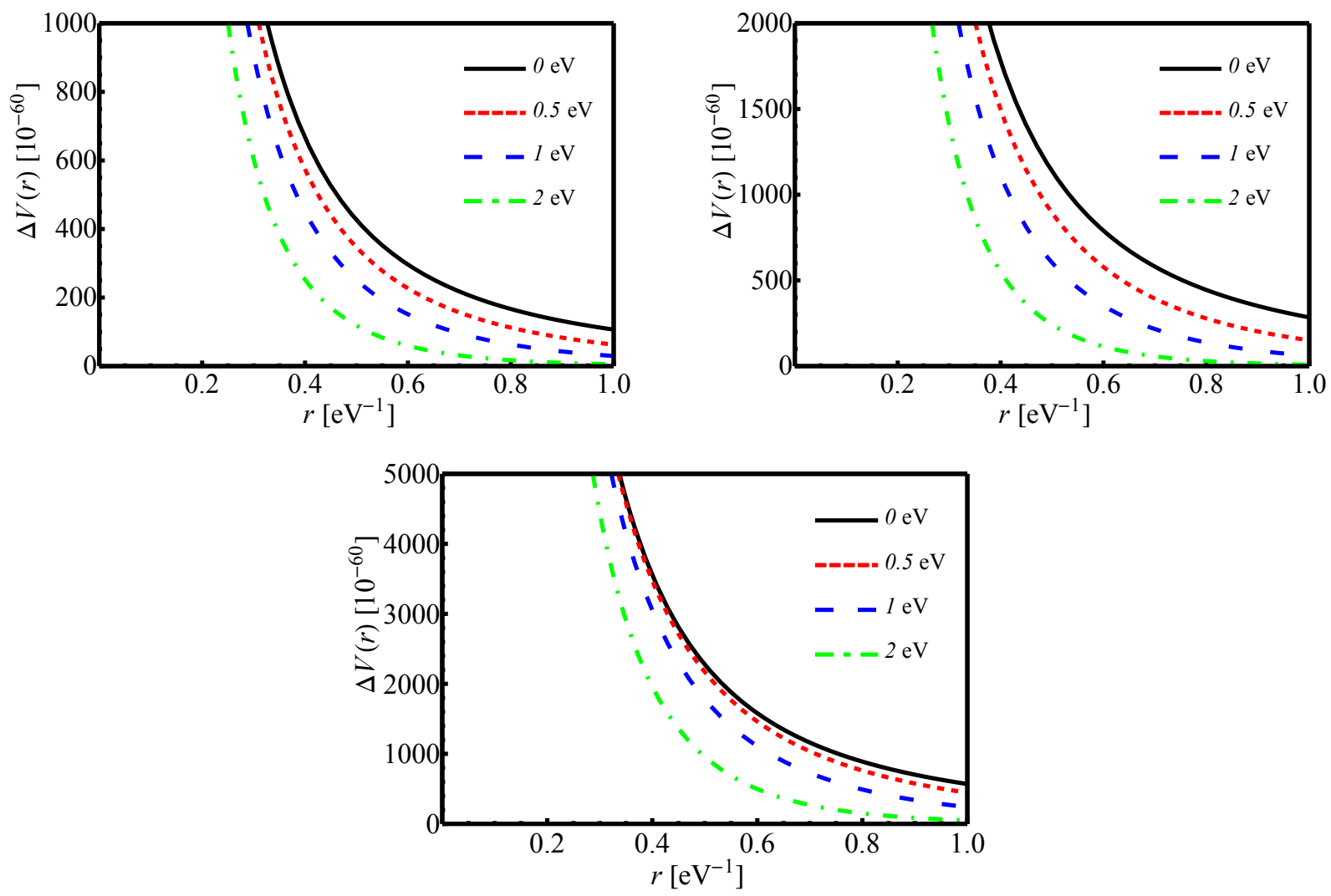

FIG. 9: Estimates of the branch-cut terms $\Delta V_{H}(r), \Delta V_{\psi}(r)$ and $\Delta V_{A}(r)$ resulting from scalar, fermion and gauge fields, as functions of the distance $r$, are shown in the upper left, upper right and lower pannels, respectively.

In the opposite limit where $\hat{r}_{H, \psi, A} \ll 1$, we find respectively for the scalar, Dirac fermion and gauge-boson contributions to $\Delta V(r)$ that

$$
\begin{aligned}
\Delta V_{H}(r) & =\frac{G}{20 \pi r^{2}}+\frac{G m_{H}^{2}}{6 \pi}\left(\ln \left(m_{H} r\right)+\gamma_{E}+\frac{1}{3}\right)+\mathcal{O}(\hat{r}) \\
\Delta V_{\psi}(r) & =\frac{G}{15 \pi r^{2}}+\frac{G m_{\psi}^{2}}{3 \pi}\left(\ln \left(m_{\psi} r\right)+\gamma_{E}-\frac{2}{3}\right)+\mathcal{O}(\hat{r}) \\
\Delta V_{A}(r) & =\frac{4 G}{15 \pi r^{2}}-\frac{2 G m_{A}^{2}}{3 \pi}\left(\ln \left(m_{A} r\right)+\gamma_{E}-\frac{25}{12}\right)+\mathcal{O}(\hat{r}) .
\end{aligned}
$$

In the above small- $\hat{r}$ expansion, the first term on the RHS of the above equations represents the correction to the potential assuming that the particle in the loop is strictly massless. These leading terms are consistent with the ones presented in the literature [26, 30, 34, 35]. For massive fields, however, the relevant subleading correction to $\Delta V(r)$ is logarithmically enhanced in $r$, as long as $r \ll 1 / 2 m$.

\section{CONCLUSIONS}

We have revisited the calculation of matter quantum effects on the graviton self-energy, assuming a flat Minkowski background metric. One of the central goals of our study has been to obtain a deeper understanding of the underlying mechanism that renders the graviton massless. To this end, we have first considered a gauged Abelian Higgs model, which has been quantized within the framework of the background field method. After writing down the respective diffeomorphically invariant path integral, we have derived a master Ward identity for the path integral as a consequence of its invariance under diffeomorphisms. This Ward identity does not ensure by itself the transversality of the graviton self-energy. The latter property of masslessness of the graviton is only obtained upon imposing minimization conditions to the effective action. In this respect, we have found that the minimization of the effective action is strongly related 
with the renormalization of the cosmological constant $\Lambda$, and this relation can be enforced to all orders in perturbation theory, by means of a Graviton Low-Energy Theorem (GLET), which we derived in this paper.

In the context of the Abelian Higgs model mentioned above, we have also calculated the matter quantum corrections to the Newtonian potential. As we have not considered graviton quantum loop effects in our study, it is evident that matter contributions to the graviton self-energy are independent of the gauge fixing parameters $\xi_{D}$ and $\sigma$ of the diffeomorphisms. In our calculations, however, the gauge dependence due to diffeomorphisms does formally enter when considering the resummed graviton propagator. Nevertheless, when calculating the S-matrix amplitude for the scattering of two scalar fields, this background gauge dependence is removed by virtue of the Ward identity derived in Section [II and the fact that the gravitationally scattered particles are on their mass shell. Hence, the analytic results we have presented in this article are diffeomorphisms invariant. On the other hand, gauge-boson loops have been calculated in the Feynman-'t Hooft gauge $\xi_{G}=1$. Since S-matrix elements are independent of the gauge-fixing parameter $\xi_{G}$, the graviton self-energy is expected to be independent of $\xi_{G}$ as well, especially when considering the elastic gravitational scattering of two gauge-singlet scalars. As a consequence, we expect that the Newtonian potential $V(r)$ will not depend on the gauge-fixing parameter $\xi_{G}$.

Treating quantum gravity as an effective field theory, we have presented analytical formulae for matter quantum effects on the Newtonian potential $V(r)$, in terms of modified Bessel and Struve functions which depend on the particle masses in the loop. Thus, we have found that the corrections to $V(r)$ exhibit an exponential fall-off dependence on the distance $r$, once the non-relativistic limit with respect to the non-zero loop masses is properly taken into account. In the massless limit of scalars, fermions and gauge bosons in the loops, we recover the well-known results that have been presented in the literature.

Like the well-known Higgs-boson low-energy theorem that holds in particle-physics models, such as the Standard Model, the GLET is a very powerful theorem. As was explicitly shown in this paper, both the GLET (III.50) and the diffeomorphisms WI (II.34) are required to forbid the appearance of a mass for the graviton field, which might be induced by quantum-loop effects. We have derived the GLET for a flat geometry, where a global shift symmetry between the background graviton field and the Minkowski metric exists. Given the property of background independence of the background field method, we expect to be able to extend this theorem to general curved background metrics. However, such a generalization is beyond the scope of the present paper. We hope to be able to report progress on this issue in a forthcoming communication.

\section{Acknowledgements}

We would like to thank Luis Alvarez-Gaume for discussions concerning the topic of the graviton mass in theories of quantum gravity. The work D.B. is supported by an STFC PhD studentship, and the work of A.P. is supported by the Lancaster-Manchester-Sheffield Consortium for Fundamental Physics under STFC grant ST/L000520/1. 


\section{Appendix A: Feynman Rules}

In this appendix we list all relevant Feynman rules which have been used in our calculations. We define all momenta as outgoing from the vertex, obeying energy-momentum conservation.

\section{Graviton Propagator}

Since our computations pertain to gauge-invariant S-matrix amplitudes, we employ the simplified form of the graviton propagator in the harmonic gauge, which is given by the RHS of V.4. For this choice of gauge, the diffeomorphisms gauge-fixing parameters $\xi_{D}$ and $\sigma$ take on the values: $\xi_{D}=1, \sigma=1 / 2$. For completeness, however, we present the general expression for the tree-level graviton propagator for arbitrary gauge parameters $\xi_{D}$ and $\sigma$ :

$$
\begin{aligned}
W W W W W= & \frac{1}{p^{2}+i \epsilon}\left[P^{\mu \nu \rho \sigma}-\left(4\left(1+\xi_{D}\right)+\frac{8}{\sigma-1}+\frac{3-\xi_{D}}{(\sigma-1)^{2}}\right) \frac{p^{\mu} p^{\nu} p^{\rho} p^{\sigma}}{\left(p^{2}\right)^{2}}\right. \\
& +\left(2+\frac{1}{\sigma-1}\right)\left(\frac{p^{\mu} p^{\nu}}{p^{2}} \eta^{\rho \sigma}+\frac{p^{\rho} p^{\sigma}}{p^{2}} \eta^{\mu \nu}\right) \\
& \left.+\left(\xi_{D}-1\right)\left(\frac{p^{\mu} p^{\rho}}{p^{2}} \eta^{\nu \sigma}+\frac{p^{\mu} p^{\sigma}}{p^{2}} \eta^{\nu \rho}+\frac{p^{\nu} p^{\rho}}{p^{2}} \eta^{\mu \sigma}+\frac{p^{\nu} p^{\sigma}}{p^{2}} \eta^{\mu \rho}\right)\right]
\end{aligned}
$$

with $P^{\mu \nu \rho \sigma}=\eta^{\mu \rho} \eta^{\nu \sigma}+\eta^{\mu \rho} \eta^{\nu \sigma}-\eta^{\mu \nu} \eta^{\rho \sigma}$.

\section{Graviton-Scalar-Scalar Vertex}

The coupling for the scalar-scalar-graviton vertex $H(p)-H(q)-h^{\mu \nu}(l)$ reads:

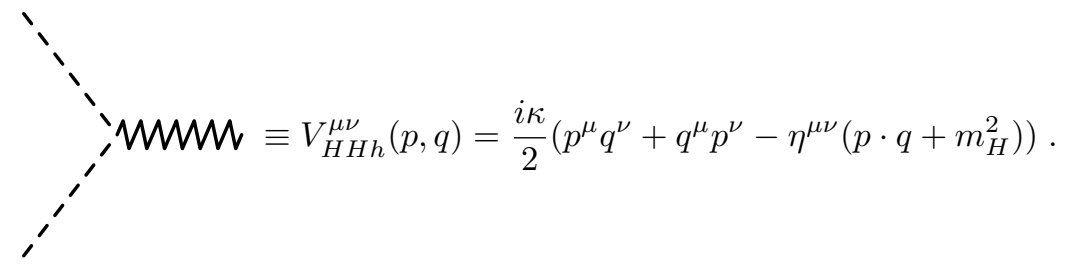

\section{Graviton-Graviton-Scalar-Scalar Vertex}

The quartic coupling $H(p)-H(q)-h^{\mu \nu}(l)-h^{\rho \sigma}(k)$ is given by

$$
\begin{aligned}
& \therefore \sqrt{2}=V_{H+\rho h h}^{\mu \nu, \rho \sigma}(p, q) \\
& =i \kappa^{2}\left(\left[\frac{1}{4}\left(\eta^{\mu \nu} I^{\rho \sigma \alpha \beta}+\eta^{\rho \sigma} I^{\mu \nu \alpha \beta}\right)-I_{\delta}^{\mu \nu \alpha} I^{\rho \sigma \beta \delta}\right]\left(p_{\alpha} q_{\beta}+q_{\alpha} p_{\beta}\right)\right. \\
& \left.+\frac{1}{2}\left(I^{\mu \nu \rho \sigma}-\frac{1}{2} \eta^{\mu \nu} \eta^{\rho \sigma}\right)\left[\left(p \cdot q+m_{H}^{2}\right)\right]\right)
\end{aligned}
$$

where

$$
I^{\mu \nu \rho \sigma} \equiv \frac{1}{2}\left(\eta^{\mu \rho} \eta^{\nu \sigma}+\eta^{\mu \sigma} \eta^{\nu \rho}\right)
$$


Note that the quartic coupling $H(p)-H(q)-h^{\mu \nu}(l)-h^{\rho \sigma}(k)$ only depends on the four-momenta $p$ and $q$ of the scalar particles.

\section{Graviton-Fermion-Fermion Vertex}

The fermion-fermion-graviton interaction $\bar{\psi}(p)-\psi(q)-h^{\mu \nu}(l)$ is given by

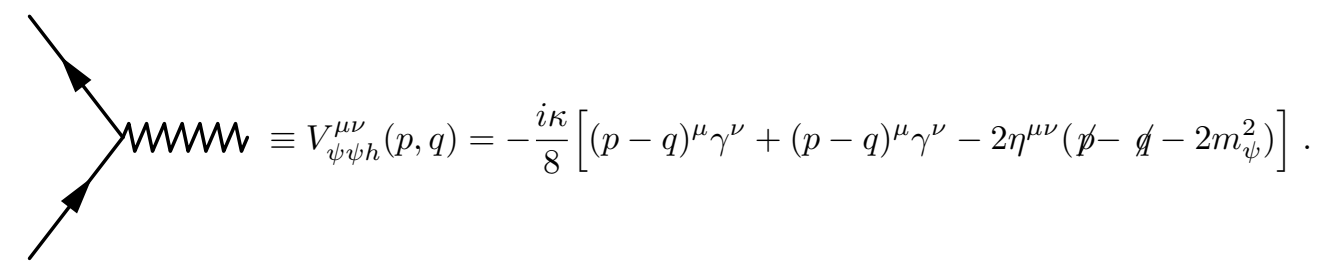

\section{Graviton-Graviton-Fermion-Fermion Coupling}

The fermion-fermion-graviton-graviton interaction $\bar{\psi}(p)-\psi(q)-h^{\mu \nu}(l)-h^{\rho \sigma}(k)$ reads:

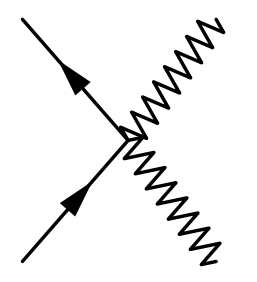

$$
\equiv V_{\psi \psi h h}^{\mu \nu, \rho \sigma}(p, q)
$$$$
=i \frac{\kappa^{2}}{8}\left[\frac { 1 } { 4 } \left(\left[\eta^{\mu \nu} \gamma^{\rho}\left(p^{\sigma}-q^{\sigma}\right)+\eta^{\rho \sigma} \gamma^{\mu}\left(p^{\nu}-q^{\nu}\right)+\frac{3}{2} \eta^{\nu \rho} \gamma^{\mu}\left(p^{\sigma}-q^{\sigma}\right)\right.\right.\right.
$$$$
\left.\left.+\frac{3}{2} \eta^{\nu \rho} \gamma^{\sigma}\left(p^{\mu}-q^{\mu}\right)+(\mu \leftrightarrow \nu)\right]+(\rho \leftrightarrow \sigma)\right)
$$$$
\left.-\frac{1}{2}\left(I^{\mu \nu \rho \sigma}-\frac{1}{2} \eta^{\mu \nu} \eta^{\rho \sigma}\right)\left[\left(\not p-\not q-2 m_{\psi}^{2}\right)\right]\right] \text {. }
$$

Like in the scalar case, the quartic coupling $\bar{\psi}(p)-\psi(q)-h^{\mu \nu}(l)-h^{\rho \sigma}(k)$ only depends on the four-momenta $p$ and $q$ of the fermion particles.

\section{Graviton-Gauge-Gauge Vertex}

The interaction vertex involving two gauge bosons $A^{\rho}(p)$ and $A^{\sigma}(q)$ and one graviton $h^{\mu \nu}(l)$ is given by

$$
\begin{aligned}
3 \text { SuW } \equiv & V_{A A h}^{\rho, \sigma, \mu \nu}(p, q) \\
= & -\frac{i \kappa}{2}\left[\left(p \cdot q+m_{A}^{2}\right)\left(2 I^{\mu \nu \rho \sigma}-\eta^{\mu \nu} \eta^{\rho \sigma}\right)\right. \\
& +\eta^{\mu \nu} p^{\sigma} q^{\rho}-\left(\eta^{\mu \sigma} p^{\nu} q^{\rho}+\eta^{\mu \sigma} p^{\nu} q^{\rho}-\eta^{\rho \sigma} p^{\mu} q^{\nu}+(\mu \leftrightarrow \nu)\right) \\
& \left.+\frac{1}{\xi_{G}}\left(\eta^{\mu \nu}\left(p^{\rho} p^{\sigma}+p^{\rho} q^{\sigma}+q^{\rho} q^{\sigma}\right)-\left[\eta^{\nu \sigma} p^{\mu} p^{\rho}+\eta^{\nu \rho} q^{\mu} q^{\sigma}+(\mu \leftrightarrow \nu)\right]\right)\right] .
\end{aligned}
$$




\section{Graviton-Graviton-Gauge-Gauge Quartic Coupling}

The quartic coupling involving two gauge bosons $A^{\alpha}(p)$ and $A^{\beta}(q)$ and two gravitons $h^{\mu \nu}(l)$ and $h^{\rho \sigma}(k)$ is found to be

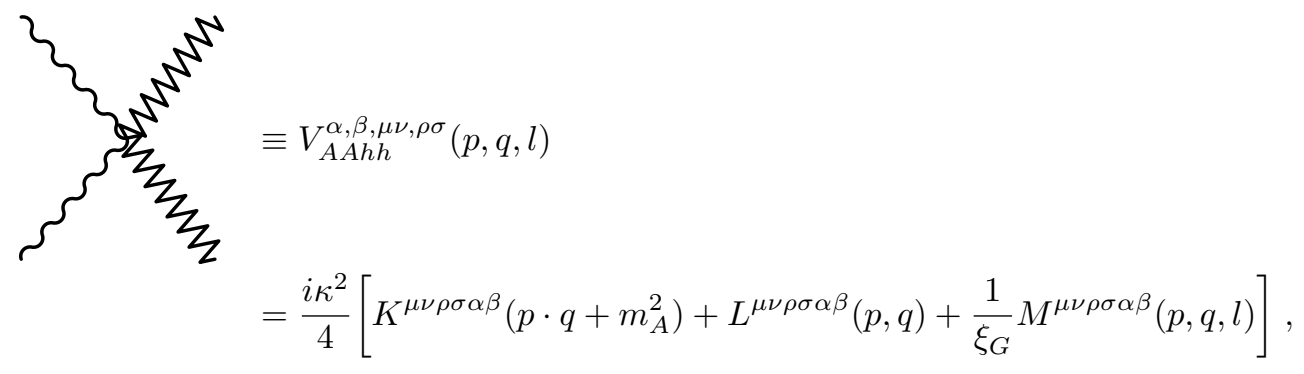

where

$$
\begin{aligned}
& L^{\mu \nu \rho \sigma \alpha \beta}(p, q)= \\
& \eta^{\nu \rho} \eta^{\mu \sigma} p^{\beta} q^{\alpha}+\eta^{\mu \rho} \eta^{\nu \sigma} p^{\beta} q^{\alpha}+\eta^{\beta \nu} \eta^{\rho \sigma} p^{\mu} q^{\alpha}-\eta^{\beta \sigma} \eta^{\mu \rho} p^{\nu} q^{\alpha}-\eta^{\beta \rho} \eta^{\mu \sigma} p^{\nu} q^{\alpha}+\eta^{\beta \mu} \eta^{\rho \sigma} p^{\nu} q^{\alpha}+\eta^{\mu \nu} \eta^{\beta \sigma} p^{\rho} q^{\alpha} \\
& -\eta^{\beta \nu} \eta^{\mu \sigma} p^{\rho} q^{\alpha}-\eta^{\beta \mu} \eta^{\nu \sigma} p^{\rho} q^{\alpha}-\eta^{\beta \sigma} p^{\mu} \eta^{\nu \rho} q^{\alpha}-\eta^{\beta \mu} p^{\sigma} \eta^{\nu \rho} q^{\alpha}+\eta^{\mu \nu} \eta^{\beta \rho} p^{\sigma} q^{\alpha}-\eta^{\beta \nu} \eta^{\mu \rho} p^{\sigma} q^{\alpha}-\eta^{\beta \rho} p^{\mu} \eta^{\nu \sigma} q^{\alpha} \\
& -p^{\beta} \eta^{\mu \nu} \eta^{\rho \sigma} q^{\alpha}+\eta^{\alpha \nu} \eta^{\rho \sigma} p^{\beta} q^{\mu}+\eta^{\beta \rho} \eta^{\alpha \sigma} p^{\nu} q^{\mu}+\eta^{\alpha \rho} \eta^{\beta \sigma} p^{\nu} q^{\mu}-\eta^{\alpha \nu} \eta^{\beta \sigma} p^{\rho} q^{\mu}+\eta^{\alpha \beta} \eta^{\nu \sigma} p^{\rho} q^{\mu}-\eta^{\alpha \sigma} p^{\beta} \eta^{\nu \rho} q^{\mu} \\
& -\eta^{\alpha \nu} \eta^{\beta \rho} p^{\sigma} q^{\mu}+\eta^{\alpha \beta} \eta^{\nu \rho} p^{\sigma} q^{\mu}-\eta^{\alpha \rho} p^{\beta} \eta^{\nu \sigma} q^{\mu}-\eta^{\alpha \beta} p^{\nu} \eta^{\rho \sigma} q^{\mu}+\eta^{\alpha \mu} \eta^{\rho \sigma} p^{\beta} q^{\nu}+\eta^{\beta \rho} \eta^{\alpha \sigma} p^{\mu} q^{\nu}+\eta^{\alpha \rho} \eta^{\beta \sigma} p^{\mu} q^{\nu} \\
& -\eta^{\alpha \mu} \eta^{\beta \sigma} p^{\rho} q^{\nu}+\eta^{\alpha \beta} \eta^{\mu \sigma} p^{\rho} q^{\nu}-\eta^{\alpha \sigma} p^{\beta} \eta^{\mu \rho} q^{\nu}-\eta^{\alpha \mu} \eta^{\beta \rho} p^{\sigma} q^{\nu}+\eta^{\alpha \beta} \eta^{\mu \rho} p^{\sigma} q^{\nu}-\eta^{\alpha \rho} p^{\beta} \eta^{\mu \sigma} q^{\nu}-\eta^{\alpha \beta} p^{\mu} \eta^{\rho \sigma} q^{\nu} \\
& +\eta^{\mu \nu} \eta^{\alpha \sigma} p^{\beta} q^{\rho}-\eta^{\alpha \sigma} \eta^{\beta \nu} p^{\mu} q^{\rho}+\eta^{\alpha \beta} \eta^{\nu \sigma} p^{\mu} q^{\rho}-\eta^{\alpha \sigma} \eta^{\beta \mu} p^{\nu} q^{\rho}+\eta^{\alpha \beta} \eta^{\mu \sigma} p^{\nu} q^{\rho}+\eta^{\beta \mu} \eta^{\alpha \nu} p^{\sigma} q^{\rho}+\eta^{\alpha \mu} \eta^{\beta \nu} p^{\sigma} q^{\rho} \\
& -\eta^{\alpha \beta} \eta^{\mu \nu} p^{\sigma} q^{\rho}-\eta^{\alpha \nu} p^{\beta} \eta^{\mu \sigma} q^{\rho}-\eta^{\alpha \mu} p^{\beta} \eta^{\nu \sigma} q^{\rho}+\eta^{\mu \nu} \eta^{\alpha \rho} p^{\beta} q^{\sigma}-\eta^{\alpha \rho} \eta^{\beta \nu} p^{\mu} q^{\sigma}+\eta^{\alpha \beta} \eta^{\nu \rho} p^{\mu} q^{\sigma}-\eta^{\alpha \rho} \eta^{\beta \mu} p^{\nu} q^{\sigma} \\
& +\eta^{\alpha \beta} \eta^{\mu \rho} p^{\nu} q^{\sigma}+\eta^{\beta \mu} \eta^{\alpha \nu} p^{\rho} q^{\sigma}+\eta^{\alpha \mu} \eta^{\beta \nu} p^{\rho} q^{\sigma}-\eta^{\alpha \beta} \eta^{\mu \nu} p^{\rho} q^{\sigma}-\eta^{\alpha \nu} p^{\beta} \eta^{\mu \rho} q^{\sigma}-\eta^{\alpha \mu} p^{\beta} \eta^{\nu \rho} q^{\sigma},
\end{aligned}
$$

$K^{\mu \nu \rho \sigma \alpha \beta}=$

$\eta^{\alpha \mu} \eta^{\beta \rho} \eta^{\nu \sigma}+\eta^{\alpha \beta} \eta^{\mu \nu} \eta^{\rho \sigma}+\eta^{\alpha \sigma} \eta^{\beta \nu} \eta^{\mu \rho}+\eta^{\alpha \nu} \eta^{\beta \sigma} \eta^{\mu \rho}+\eta^{\alpha \rho} \eta^{\beta \nu} \eta^{\mu \sigma}+\eta^{\alpha \nu} \eta^{\beta \rho} \eta^{\mu \sigma}+\eta^{\alpha \sigma} \eta^{\beta \mu} \eta^{\nu \rho}+\eta^{\alpha \mu} \eta^{\beta \sigma} \eta^{\nu \rho}$ $+\eta^{\alpha \rho} \eta^{\beta \mu} \eta^{\nu \sigma}-\eta^{\alpha \beta} \eta^{\sigma \mu} \eta^{\nu \rho}-\eta^{\alpha \beta} \eta^{\mu \rho} \eta^{\nu \sigma}-\eta^{\alpha \nu} \eta^{\beta \mu} \eta^{\rho \sigma}-\eta^{\alpha \mu} \eta^{\beta \nu} \eta^{\rho \sigma}-\eta^{\alpha \sigma} \eta^{\beta \rho} \eta^{\mu \nu}-\eta^{\alpha \rho} \eta^{\beta \sigma} \eta^{\mu \nu}$

$$
\begin{aligned}
& M^{\mu \nu \rho \sigma \alpha \beta}(p, q, l)= \\
& \eta^{\nu \rho} \eta^{\mu \sigma} p^{\beta} p^{\alpha}+\eta^{\mu \rho} \eta^{\nu \sigma} p^{\beta} p^{\alpha}+\eta^{\nu \rho} \eta^{\mu \sigma} q^{\beta} p^{\alpha}+\eta^{\mu \rho} \eta^{\nu \sigma} q^{\beta} p^{\alpha}+\eta^{\beta \nu} \eta^{\rho \sigma} p^{\mu} p^{\alpha}+\eta^{\beta \nu} \eta^{\rho \sigma} q^{\mu} p^{\alpha}+\eta^{\beta \nu} \eta^{\rho \sigma} l^{\mu} p^{\alpha} \\
& -\eta^{\beta \sigma} \eta^{\mu \rho} p^{\nu} p^{\alpha}-\eta^{\beta \rho} \eta^{\mu \sigma} p^{\nu} p^{\alpha}+\eta^{\beta \mu} \eta^{\rho \sigma} p^{\nu} p^{\alpha}+\eta^{\beta \mu} \eta^{\rho \sigma} q^{\nu} p^{\alpha}+\eta^{\beta \mu} \eta^{\rho \sigma} l^{\nu} p^{\alpha}+\eta^{\mu \nu} \eta^{\beta \sigma} p^{\rho} p^{\alpha}-\eta^{\beta \nu} \eta^{\mu \sigma} p^{\rho} p^{\alpha} \\
& -\eta^{\beta \mu} \eta^{\nu \sigma} p^{\rho} p^{\alpha}-\eta^{\beta \sigma} p^{\mu} \eta^{\nu \rho} p^{\alpha}-\eta^{\beta \mu} p^{\sigma} \eta^{\nu \rho} p^{\alpha}+\eta^{\mu \nu} \eta^{\beta \rho} p^{\sigma} p^{\alpha}-\eta^{\beta \nu} \eta^{\mu \rho} p^{\sigma} p^{\alpha}-\eta^{\beta \rho} p^{\mu} \eta^{\nu \sigma} p^{\alpha}-p^{\beta} \eta^{\mu \nu} \eta^{\rho \sigma} p^{\alpha} \\
& -q^{\beta} \eta^{\mu \nu} \eta^{\rho \sigma} p^{\alpha}-l^{\beta} \eta^{\mu \nu} \eta^{\rho \sigma} p^{\alpha}+\eta^{\alpha \nu} \eta^{\rho \sigma} p^{\mu} p^{\beta}+\eta^{\alpha \nu} \eta^{\rho \sigma} l^{\mu} p^{\beta}+\eta^{\alpha \mu} \eta^{\rho \sigma} p^{\nu} p^{\beta}+\eta^{\alpha \mu} \eta^{\rho \sigma} l^{\nu} p^{\beta}-l^{\alpha} \eta^{\mu \nu} \eta^{\rho \sigma} p^{\beta} \\
& +\eta^{\alpha \nu} \eta^{\rho \sigma} q^{\beta} p^{\mu}+\eta^{\alpha \nu} \eta^{\rho \sigma} l^{\beta} p^{\mu}-\eta^{\alpha \nu} \eta^{\beta \sigma} p^{\rho} p^{\mu}-\eta^{\alpha \nu} \eta^{\beta \sigma} l^{\rho} p^{\mu}-\eta^{\alpha \nu} \eta^{\beta \rho} p^{\sigma} p^{\mu}-\eta^{\alpha \nu} \eta^{\beta \rho} l^{\sigma} p^{\mu}+\eta^{\alpha \mu} \eta^{\rho \sigma} q^{\beta} p^{\nu} \\
& +\eta^{\alpha \mu} \eta^{\rho \sigma} l^{\beta} p^{\nu}-\eta^{\alpha \mu} \eta^{\beta \sigma} p^{\rho} p^{\nu}-\eta^{\alpha \mu} \eta^{\beta \sigma} l^{\rho} p^{\nu}-\eta^{\alpha \mu} \eta^{\beta \rho} p^{\sigma} p^{\nu}-\eta^{\alpha \mu} \eta^{\beta \rho} l^{\sigma} p^{\nu}+\eta^{\mu \nu} \eta^{\beta \sigma} l^{\alpha} p^{\rho}-\eta^{\alpha \nu} \eta^{\beta \sigma} l^{\mu} p^{\rho} \\
& -\eta^{\alpha \mu} \eta^{\beta \sigma} l^{\nu} p^{\rho}+\eta^{\mu \nu} \eta^{\beta \rho} l^{\alpha} p^{\sigma}-\eta^{\alpha \nu} \eta^{\beta \rho} l^{\mu} p^{\sigma}-\eta^{\alpha \mu} \eta^{\beta \rho} l^{\nu} p^{\sigma}+\eta^{\beta \nu} \eta^{\rho \sigma} q^{\mu} l^{\alpha}+\eta^{\beta \mu} \eta^{\rho \sigma} q^{\nu} l^{\alpha}+\eta^{\nu \rho} \eta^{\mu \sigma} q^{\alpha} q^{\beta} \\
& +\eta^{\mu \rho} \eta^{\nu \sigma} q^{\alpha} q^{\beta}+\eta^{\mu \nu} \eta^{\alpha \sigma} q^{\rho} l^{\beta}+\eta^{\mu \nu} \eta^{\alpha \rho} q^{\sigma} l^{\beta}+\eta^{\beta \nu} \eta^{\rho \sigma} q^{\alpha} q^{\mu}+\eta^{\alpha \nu} \eta^{\rho \sigma} q^{\beta} q^{\mu}+\eta^{\beta \nu} \eta^{\rho \sigma} q^{\alpha} l^{\mu}+\eta^{\beta \nu} \eta^{\rho \sigma} l^{\alpha} l^{\mu} \\
& +\eta^{\alpha \nu} \eta^{\rho \sigma} q^{\beta} l^{\mu}+\eta^{\alpha \nu} \eta^{\rho \sigma} l^{\beta} l^{\mu}+\eta^{\beta \mu} \eta^{\rho \sigma} q^{\alpha} q^{\nu}+\eta^{\alpha \mu} \eta^{\rho \sigma} q^{\beta} q^{\nu}-\eta^{\alpha \sigma} q^{\beta} \eta^{\mu \rho} q^{\nu}-\eta^{\alpha \rho} q^{\beta} \eta^{\mu \sigma} q^{\nu}+\eta^{\beta \mu} \eta^{\rho \sigma} q^{\alpha} l^{\nu} \\
& +\eta^{\beta \mu} \eta^{\rho \sigma} l^{\alpha} l^{\nu}+\eta^{\alpha \mu} \eta^{\rho \sigma} q^{\beta} l^{\nu}+\eta^{\alpha \mu} \eta^{\rho \sigma} l^{\beta} l^{\nu}+\eta^{\mu \nu} \eta^{\alpha \sigma} q^{\beta} q^{\rho}-\eta^{\alpha \sigma} \eta^{\beta \nu} q^{\mu} q^{\rho}-\eta^{\alpha \sigma} \eta^{\beta \nu} l^{\mu} q^{\rho}-\eta^{\alpha \sigma} \eta^{\beta \mu} q^{\nu} q^{\rho} \\
& -\eta^{\alpha \sigma} \eta^{\beta \mu} l^{\nu} q^{\rho}-\eta^{\alpha \nu} q^{\beta} \eta^{\mu \sigma} q^{\rho}-\eta^{\alpha \mu} q^{\beta} \eta^{\nu \sigma} q^{\rho}+\eta^{\mu \nu} \eta^{\beta \sigma} l^{\alpha} l^{\rho}+\eta^{\mu \nu} \eta^{\alpha \sigma} l^{\beta} l^{\rho}-\eta^{\alpha \sigma} \eta^{\beta \nu} q^{\mu} l^{\rho}-\eta^{\alpha \sigma} \eta^{\beta \nu} l^{\mu} l^{\rho} \\
& -\eta^{\alpha \nu} \eta^{\beta \sigma} l^{\mu} l^{\rho}-\eta^{\alpha \sigma} \eta^{\beta \mu} q^{\nu} l^{\rho}-\eta^{\alpha \sigma} \eta^{\beta \mu} l^{\nu} l^{\rho}-\eta^{\alpha \mu} \eta^{\beta \sigma} l^{\nu} l^{\rho}-\eta^{\alpha \sigma} q^{\beta} q^{\mu} \eta^{\nu \rho}-\eta^{\alpha \mu} q^{\beta} q^{\sigma} \eta^{\nu \rho}+\eta^{\mu \nu} \eta^{\alpha \rho} q^{\beta} q^{\sigma} \\
& -\eta^{\alpha \rho} \eta^{\beta \nu} q^{\mu} q^{\sigma}-\eta^{\alpha \rho} \eta^{\beta \nu} l^{\mu} q^{\sigma}-\eta^{\alpha \rho} \eta^{\beta \mu} q^{\nu} q^{\sigma}-\eta^{\alpha \rho} \eta^{\beta \mu} l^{\nu} q^{\sigma}-\eta^{\alpha \nu} q^{\beta} \eta^{\mu \rho} q^{\sigma}+\eta^{\mu \nu} \eta^{\beta \rho} l^{\alpha} l^{\sigma}+\eta^{\mu \nu} \eta^{\alpha \rho} l^{\beta} l^{\sigma} \\
& -\eta^{\alpha \rho} \eta^{\beta \nu} q^{\mu} l^{\sigma}-\eta^{\alpha \rho} \eta^{\beta \nu} l^{\mu} l^{\sigma}-\eta^{\alpha \nu} \eta^{\beta \rho} l^{\mu} l^{\sigma}-\eta^{\alpha \rho} \eta^{\beta \mu} q^{\nu} l^{\sigma}-\eta^{\alpha \rho} \eta^{\beta \mu} l^{\nu} l^{\sigma}-\eta^{\alpha \mu} \eta^{\beta \rho} l^{\nu} l^{\sigma}-\eta^{\alpha \rho} q^{\beta} q^{\mu} \eta^{\nu \sigma} \\
& -q^{\alpha} q^{\beta} \eta^{\mu \nu} \eta^{\rho \sigma}-l^{\alpha} q^{\beta} \eta^{\mu \nu} \eta^{\rho \sigma}-q^{\alpha} l^{\beta} \eta^{\mu \nu} \eta^{\rho \sigma}-2 l^{\alpha} l^{\beta} \eta^{\mu \nu} \eta^{\rho \sigma} \text {. }
\end{aligned}
$$


[1] B. DeWitt, Phys. Rev. 162, 1195 (1967).

[2] B. DeWitt, Phys. Rev. 162, 1239 (1967).

[3] M. Gell-Mann, R. J. Oakes and B. Renner, Phys. Rev. 175, 2195 (1968).

[4] J. Goldstone, Nuovo Cim. 19, 154 (1961).

[5] D. V. Volkov and V. P. Akulov, Phys. Lett. B 46, 109 (1973).

[6] J. Wess and B. Zumino, Nucl. Phys. B 70, 39 (1974).

[7] S. R. Coleman and E. J. Weinberg, Phys. Rev. D 7, 1888 (1973).

[8] E. Gildener and S. Weinberg, Phys. Rev. D 13, 3333 (1976).

[9] H. E. Haber and G. L. Kane, Phys. Rept. 117 (1985) 75.

[10] S. P. Martin, Adv. Ser. Direct. High Energy Phys. 21, 1 (2010) hep-ph/9709356.

[11] T. Ibrahim and P. Nath, Rev. Mod. Phys. 80 (2008) 577 arXiv:0705.2008 [hep-ph]].

[12] D. M. Capper, Il Nuovo Cimento A 25, 29 (1975).

[13] D. Capper and M. Duff, Nucl. Phys. B82, 147 (1974).

[14] D. Capper, M. Duff and L. Halpern, Phys. Rev. D 10, 461 (1974).

[15] D. Capper, G. Leibbrandt and M. Medrano, Phys. Rev. D 8, 4320 (1973).

[16] D. Capper, J. Dulwich and M. Medrano, Nucl. Phys. B254, 737 (1985).

[17] G. Gabadadze and A. Gruzinov, Phys. Rev. D 72, 124007 (2005), arXiv:0312074 [hep-th].

[18] M. Tegmark et al., Phys. Rev. D 69, 103501 (2004), arXiv:0310723 [astro-ph].

[19] The Planck Collaboration, arXiv:1303.5076.

[20] G. 't Hooft and M. Veltman, Annales Poincare Phys. Theor. A20, 69 (1974).

[21] S. Deser and P. van Nieuwenhuizen, Phys. Rev. D 10, 411 (1974).

[22] S. Deser and P. van Nieuwenhuizen, Phys. Rev. D 10, 401 (1974).

[23] S. Deser, H.S. Tsao and P. van Nienwenhuizen, Phys. Lett. B50, 491 (1974).

[24] J. F. Donoghue, Phys. Rev. D 50, 3874 (1994).

[25] J. Donoghue, Phys. Rev. Lett. 72 (1993).

[26] H. Hamber and S. Liu, Phys. Lett. B357, 51 (1995), arXiv:9505182 [hep-th].

[27] I. Muzinich and S. Vokos, Phys. Rev. D 52, 3472 (1995), arXiv:9501083 [hep-th].

[28] A. Akhundov, S. Bellucci and A. Shiekh, Phys. Lett. B395, 16, (1997), arXiv:9611018 [gr-qc].

[29] N. Bjerrum-Bohr, Phys. Rev. D 66, 084023 (2002), arXiv:0206236 [hep-th].

[30] N. Bjerrum-Bohr, J. Donoghue, and B. Holstein, Phys. Rev. D 66, 084033 (2002), arXiv:0206236 [hep-th].

[31] G. G. Kirilin and I. B. Khriplovich, J. Exp. Theor. Phys. 95, 981 (2002), arXiv:0207118 [gr-qc].

[32] M. Butt, Phys. Rev. D 74, 125007 (2006), arXiv:0605137 [gr-qc].

[33] S. Faller, Phys. Rev. D 77, 124039 (2008), arXiv:0708.1701

[34] M. Duff, Phys. Rev. D 9, 1837 (1974).

[35] M. Duff and J. T. Liu, Class.Quant.Grav. 18, 3207 (2001), arXiv:0003237 [hep-th].

[36] L. Alvarez-Gaume and E. Witten, Nucl. Phys. B 234, 269 (1984).

[37] G. 't Hooft and M. Veltman, Nucl. Phys. B44, 189 (1972).

[38] S. L. Adler, Phys. Rev. 177, 2426 (1969).

[39] J. S. Bell and R. Jackiw, Nuovo Cim. A 60, 47 (1969).

[40] J. Ellis, M.K. Gaillard, and D.V.Nanopulos, Nucl. Phys. B106, 292 (1976).

[41] M.A. Shifman, A.I. Vainshtein, and V.I. Zakharov, Phys. Lett. B78, 443 (1978).

[42] A.I. Vainshtein, M.B. Voloshin, V.I. Zakharov, and M.A. Shifman, Yad. Fiz. 30, 1368 (1976) [Sov. Phys. Usp. 30, 711 (1979)].

[43] S. Dawson and H.E. Haber, Int. J. Mod. Phys. A7, 107 (1992).

[44] B.A. Kniehl and M. Spira, Z. Phys. C69 77 (1995).

[45] A. Pilaftsis, Phys. Lett. B422, 201 (1998), arXiv:9711420 [hep-ph].

[46] N.K. Nielsen, Nucl. Phys. B97, 527 (1975); Nucl. Phys. B101, 173 (1975).

[47] W. Bardeen, A. Buras, D. Duke and T. Muta, Phys. Rev. D 18, 3998 (1978).

[48] H.E. De Meyer, Lett.Nuovo Cim. 11, 498 (1974). 